TRANSACTIONS OF THE

AMERICAN MATHEMATICAL SOCIETY

Volume 362, Number 10, October 2010, Pages 5501-5527

S 0002-9947(2010)05131-1

Article electronically published on May 20, 2010

\title{
HOMOLOGY GROUPS OF SYMMETRIC QUANDLES AND COCYCLE INVARIANTS OF LINKS AND SURFACE-LINKS
}

\author{
SEIICHI KAMADA AND KANAKO OSHIRO
}

\begin{abstract}
We introduce the notion of a quandle with a good involution and its homology groups. Carter et al. defined quandle cocycle invariants for oriented links and oriented surface-links. By use of good involutions, quandle cocyle invariants can be defined for links and surface-links which are not necessarily oriented or orientable. The invariants can be used in order to estimate the minimal triple point numbers of non-orientable surface-links.
\end{abstract}

\section{INTRODUCTION}

The fundamental quandles are strong invariants of links, which are generalized to any codimension-two oriented manifold pairs [9, 13, 18. Carter et al. 4 defined homology groups of quandles, which are deeply related to homology groups of racks due to Fenn et al. [10]; cf. [5, 17]. Using cocycles $\theta$ of quandles, Carter et al. [4] defined invariants of links in 3-space or surface-links in 4-space, called quandle cocycle invariants and denoted by $\Phi_{\theta}^{\text {ori }}$ in this paper. For details on quandle homologies and invariants, refer to [1, 3, 4, 5, 10, 11, 17, 21, 22, Quandle cocyle invariants can distinguish the left-handed trefoil from the right-handed trefoil $([21,22])$ and the 2-twist-spun trefoil from its copy with the opposite orientation ([2, 4, 12, 21, 22] ), although the fundamental quandles themselves cannot. In order to define quandle cocycle invariants, it is essential that links or surface-links are oriented. In this paper we introduce the notion of a quandle with a good involution, which is called a symmetric quandle, and its homology groups. This notion enables us to consider quandle cocycle invariants, denoted by $\Phi_{\theta}$, for links or surface-links which are not necessarily oriented or orientable.

For a given symmetric quandle 2 -cocycle $\theta$, we define a quandle cocycle invariant $\Phi_{\theta}$ of an unoriented link. We show that $\Phi_{\theta}$ is equal to the quandle cocycle invariant $\Phi_{\theta}^{\text {ori }}$ of an oriented link when we give an orientation to the link arbitrarily (Theorem 6.7). As a consequence, we see that $\Phi_{\theta}^{\text {ori }}$ does not depend on the orientation of a link if $\theta$ is a quandle 2-cocycle cohomologous to a symmetric quandle 2-cocycle. We can also use the fact $\Phi_{\theta}^{\text {ori }}=\Phi_{\theta}$ for calculation of $\Phi_{\theta}^{\text {ori }}$, since the calculation of $\Phi_{\theta}$ is sometimes simpler than that of $\Phi_{\theta}^{\text {ori }}$ as seen in Example 7.4

Received by the editors February 18, 2009.

2010 Mathematics Subject Classification. Primary 57M25, 57Q45; Secondary 55N99, 18 G99.

Key words and phrases. Knot, link, surface-link, knotted surface, quandle, rack, quandle homology, symmetric quandle, good involution.

The first author's research was partially supported by Grant-in-Aid for Scientific Research, JSPS.

The second author's research was partially supported by Grant-in-Aid for JSPS Research Fellowships for Young Scientists.

(C)2010 American Mathematical Society 5501 
For a given symmetric quandle 3 -cocycle $\theta$, we define a quandle cocycle invariant $\Phi_{\theta}$ of an unoriented or non-orientable surface-link. We show that when a surfacelink is orientable, the invariant $\Phi_{\theta}$ is equal to the quandle cocycle invariant $\Phi_{\theta}^{\text {ori }}$ of an oriented surface-link when we give any orientation to the surface-link (Theorem 8.5). The invariants $\Phi_{\theta}$ are essentially new and interesting for non-orientable surfacelinks. As $\Phi_{\theta}^{\text {ori }}$ can be used to estimate the minimal triple point numbers of oriented surface-links [26, 27, we can use $\Phi_{\theta}$ to estimate those of non-orientable surface-links (Theorem 8.6. Proposition 9.4 and Theorems 9.5 and 9.6).

This paper is organized as follows: In $\$ 2$ the definition of a quandle with a good involution or a symmetric quandle is defined. In $\$ 3$ we determine good involutions of trivial quandles, dihedral quandles and keis. In $\S \$$ and 5 the definitions of the associated group and homology groups of a symmetric quandle are given. In 86 we investigate cocycle invariants $\Phi_{\theta}$ for classical links and show that $\Phi_{\theta}$ is equal to $\Phi_{\theta}^{\text {ori }}$. In $\$ 7$ a special case where $X$ is a kei and $\rho$ is the identity map of $X$ is considered. In $\$ 8$ we discuss quandle cocycle invariants for surface-links. In $\$ 9$ examples of quandle cocycle invariants and applications on the minimal triple point numbers of surface-links are given.

\section{Quandles With Good inVolutions}

A quandle [9, 13, 18, is a set $X$ with a binary operation $(x, y) \mapsto x^{y}$ satisfying the following: (Q1) For any $x \in X, x^{x}=x$, (Q2) for any $x, y \in X$, there exists a unique element $z \in X$ with $z^{y}=x$, and (Q3) for any $x, y, z \in X,\left(x^{y}\right)^{z}=\left(x^{z}\right)^{\left(y^{z}\right)}$. We denote the element $z$ given in (Q2) by $x^{y^{-1}}$.

A rack [9] is a set $X$ with a binary operation satisfying (Q2) and (Q3).

A kei [28] or an involutory quandle [13] is a quandle satisfying the fact that $\left(x^{y}\right)^{y}=x$ for any $x, y \in X$. In other words, a kei is a quandle $X$ with $x^{y}=x^{y^{-1}}$ for any $x, y \in X$.

Definition 2.1. A map $\rho: X \rightarrow X$ is a good involution [16] if it is an involution (i.e., $\rho \circ \rho=$ id) such that $\rho\left(x^{y}\right)=\rho(x)^{y}$ and $x^{\rho(y)}=x^{y^{-1}}$ for any $x, y \in X$. Such a pair $(X, \rho)$ is called a quandle with a good involution or a symmetric quandle.

A (quandle) homomorphism $f: X \rightarrow Y$ is a map such that $f\left(x^{y}\right)=f(x)^{f(y)}$ for any $x, y \in X$. A (symmetric quandle) homomorphism $f:\left(X, \rho_{X}\right) \rightarrow\left(Y, \rho_{Y}\right)$ means a map $f: X \rightarrow Y$ such that $f\left(x^{y}\right)=f(x)^{f(y)}$ and $f\left(\rho_{X}(x)\right)=\rho_{Y}(f(x))$ for any $x, y \in X$.

Example 2.2 (cf. [16). Let $G$ be a group. The conjugation quandle, denoted by $\operatorname{conj}(G)$, is $G$ with the operation $x^{y}=y^{-1} x y$. The inversion, $\operatorname{inv}(G): G \rightarrow G ; g \mapsto$ $g^{-1}$, is a good involution of $\operatorname{conj}(G)$. We call $(\operatorname{conj}(G), \operatorname{inv}(G))$ the conjugation symmetric quandle.

Example 2.3 (cf. [16). Let $X$ be a quandle and let $X_{1}$ and $X_{2}$ be two copies of $X$. For an element $x \in X$, we denote by $x_{1}$ and $x_{2}$ the corresponding elements of $X_{1}$ and $X_{2}$, respectively. Let $D(X)$ be the disjoint union of $X_{1}$ and $X_{2}$. For any elements $x, y \in X$, we put $x_{i}^{y_{1}}=\left(x^{y}\right)_{i}$ and $x_{i}^{y_{2}}=\left(x^{y^{-1}}\right)_{i}$ for $i=1,2$. Then the set $D(X)$ is a quandle. The involution $\rho: D(X) \rightarrow D(X)$ interchanging $x_{1}$ and $x_{2}$ for every $x \in X$ is a good involution.

Definition 2.4 (cf. [16]). For a codimension-two manifold pair $(W, L)$, let $\widetilde{Q}_{L}$ be the set of homotopy classes, $x=[(D, \alpha)]$, of all pairs $(D, \alpha)$, where $D$ is an oriented 
meridian disk of $L$ and $\alpha$ is a path in $W \backslash L$ starting from a point of $\partial D$ and ending at a fixed base point $* \in W \backslash L$. It is a quandle with an operation defined by

$$
\left[\left(D_{1}, \alpha_{1}\right)\right]^{\left[\left(D_{2}, \alpha_{2}\right)\right]}=\left[\left(D_{1}, \alpha_{1} \cdot \alpha_{2}^{-1} \cdot \partial D_{2} \cdot \alpha_{2}\right)\right] .
$$

An involution $\rho: \widetilde{Q}_{L} \rightarrow \widetilde{Q}_{L}$ defined by $[(D, \alpha)] \mapsto[(-D, \alpha)]$ is a good involution of $\widetilde{Q}_{L}$, where $-D$ stands for the meridian disk $D$ with the opposite orientation. The fundamental symmetric quandle of $L$ is $\left(\widetilde{Q}_{L}, \rho\right)$.

Example 2.5. If $L$ is an unknot in $\mathbb{R}^{3}$ or an unknotted 2-sphere in $\mathbb{R}^{4}$, then $\widetilde{Q}_{L}$ consists of two elements, say $x_{1}=[(D, \alpha)]$ and $x_{2}=[(-D, \alpha)]$. This is a trivial quandle (i.e., $x_{i}^{x_{j}}=x_{i}$ for $i, j \in\{1,2\}$ ), and $\rho\left(x_{1}\right)=x_{2}$. On the other hand, if $L$ is an unknotted projective plane in $\mathbb{R}^{4}$, then $\widetilde{Q}_{L}$ consists of a single element $x$ with $\rho(x)=x$, where any surface ambiently isotopic to the projective planes that project to the standard cross-cap is unknotted.

When $L$ is transversely oriented (i.e., all meridian disks of $L$ are oriented coherently), we have a subquandle $Q_{L}$ of $\widetilde{Q}_{L}$ consisting of all homotopy classes of pairs $(D, \alpha)$ such that $D$ has the given orientation; cf. p. 359 of $[9]$. Then $\left(\widetilde{Q}_{L}, \rho\right)$ is isomorphic to $\left(D\left(Q_{L}\right), \rho\right)$ in the sense of Example 2.3.

\section{Good involutions of TRIVIAL QUANDLES, DIHEDRAL QUANDLES AND KEIS}

In this section we study good involutions of trivial quandles, dihedral quandles and keis.

A trivial quandle is a quandle whose operation is trivial, i.e., $x^{y}=x$ for any $x, y \in X$. A trivial quandle consisting of $n$ elements will be denoted by $T_{n}$ later.

Proposition 3.1. If $X$ is a trivial quandle, then every involution of $X$ is a good involution. Conversely, if every involution of $X$ is a good involution, then $X$ is a trivial quandle.

Proof. Let $\rho$ be an involution of a trivial quandle $X$. For any $x, y \in X, \rho\left(x^{y}\right)=$ $\rho(x)=\rho(x)^{y}$ and $x^{\rho(y)}=x=x^{y^{-1}}$. Therefore, $\rho$ is a good involution of $X$.

Suppose that any involution of $X$ is a good involution. For any $x, y \in X$, consider an involution $\rho: X \rightarrow X$ with $\rho(x)=y, \rho(y)=x$ and $\rho(z)=z$ for $z \neq x, y \in X$. If $\rho$ is a good involution, then $x=\rho(y)=\rho\left(y^{y}\right)=\rho(y)^{y}=x^{y}$. Thus $X$ is a trivial quandle.

The dihedral quandle of order $n$ is $\mathbb{Z} / n \mathbb{Z}$ with the operation $x^{y}=2 y-x(\bmod n)$. We denote it by $R_{n}$. For simplicity, we denote an element $i+n \mathbb{Z}$ of $\mathbb{Z} / n \mathbb{Z}$ by $i$ for $i \in\{0,1, \cdots, n-1\}$.

Operations of the dihedral quandle $R_{n}$ correspond to inversions along axes of symmetry of a regular $n$-gon. Consider a regular $n$-gon whose vertices are named $0,1, \cdots, n-1 \in \mathbb{Z} / n \mathbb{Z}=X$ in this order. The inversion along the axis of symmetry passing through a vertex $y \in X$ induces a bijection $X \rightarrow X$ with $x \mapsto 2 y-x$ $(\bmod n)$.

Let $X$ be the dihedral quandle $R_{n}=\mathbb{Z} / n \mathbb{Z}$. The identity map is a good involution. If $n$ is an even number, say $2 m$, then a map $\rho: X \rightarrow X$ with $\rho(i)=i+m$ for all $i$ is a good involution. We call it the antipodal map. Moreover, if $n=2 m$ for some even number $m$, then $X$ has two more good involutions, say $\rho_{1}$ and $\rho_{2}$, with 
$\rho_{1}(i)=i$ for odd $i$ 's and $\rho_{1}(i)=i+m$ for even $i$ 's; and $\rho_{2}(i)=i+m$ for odd $i$ 's and $\rho_{2}(i)=i$ for even $i$ 's. We call them half-antipodal maps. See Figure 1

Theorem 3.2. Let $X$ be the dihedral quandle $R_{n}$.

(1) When $n$ is an odd number, the identity map is the only good involution.

(2) When $n=2 m$ and $m$ is an odd number, a good involution is either the identity map or the antipodal map.

(3) When $n=2 m$ and $m$ is an even number, there are four good involutions: the identity map, the antipodal map and two half-antipodal maps.
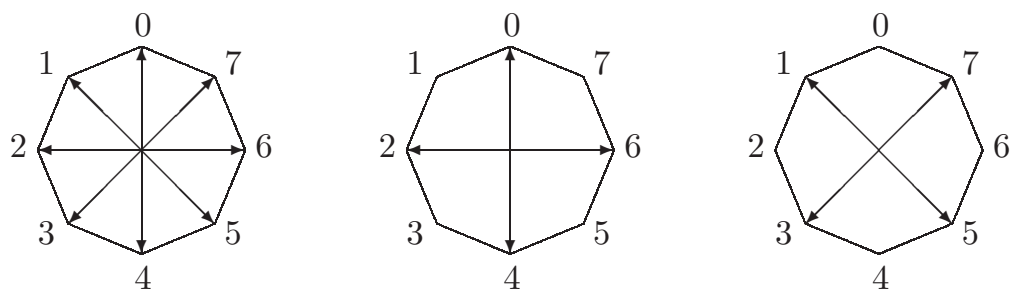

Figure 1. The antipodal map and the two half-antipodal maps

We prepare a lemma for the proof of Theorem 3.2 .

Lemma 3.3. Let $\rho$ be a good involution of $R_{n}$.

(1) For each $i \in R_{n}, \rho(i)=i$ or $\rho(i)=i+n / 2$. The latter occurs only if $n$ is even.

(2) Let $n$ be an even number. If $\rho(i)=i+n / 2$ for some $i$, then $\rho(j)=j+n / 2$ for every $j$ such that $i \equiv j(\bmod 2)$.

Proof. (1) For any $i$ and $j \in R_{n}, j^{\rho(i)}=j^{i^{-1}}=j^{i}$. Thus $2 \rho(i)=2 i$ in $\mathbb{Z} / n \mathbb{Z}$. If $n$ is odd, then $\rho(i)=i$. If $n$ is even, then $\rho(i)=i$ or $i+n / 2$.

(2) Suppose that $\rho(i)=i+n / 2$ for some $i$. Then $\rho(i+2)=\rho\left(i^{i+1}\right)=\rho(i)^{i+1}=$ $(i+n / 2)^{i+1}=i+2-n / 2=i+2+n / 2$. By induction, we have the result.

Proof of Theorem [3.2. Let $\rho$ be a good involution of $R_{n}$.

(1) When $n$ is an odd number, by Lemma $3.3(1), \rho$ is the identity map.

(2) When $n=2 m$ for an odd number $m$, suppose that $\rho$ is not the identity map. By Lemma 3.3 (1), there is an element $i \in R_{n}$ such that $\rho(i)=i+m$. Then, $\rho(i+m)=i$. One of $i$ and $i+m$ is an odd number, and the other is an even number. By Lemma $3.3(2), \rho(j)=j+m$ for every $j \in R_{n}$.

(3) When $n=2 m$ for an even number $m$, suppose that $\rho$ is not the identity map. By Lemma 3.3 (1), there is an element $i \in R_{n}$ with $\rho(i)=i+m$. By Lemma 3.3 (2), we see that $\rho$ is the antipodal or a half-antipodal map.

A good involution of a quandle is not necessarily a quandle homomorphism. It is a quandle homomorphism if and only if the quandle is a kei. A kei is characterized in terms of a good involution as follows.

Proposition 3.4. Let $X$ be a quandle. The following are equivalent:

(1) $X$ is a kei.

(2) The identity map of $X$ is a good involution.

(3) $X$ has a good involution which is a quandle homomorphism. 
(4) Any good involution of $X$ is a quandle homomorphism, and $X$ has at least one good involution.

Proof. (1) $\Rightarrow(2)$ : For the identity map $\rho, \rho\left(x^{y}\right)=\rho(x)^{y}$. Since $X$ is a kei, $x^{\rho(y)}=$ $x^{y}=x^{y^{-1}}$.

$(2) \Rightarrow(3)$ : It is obvious.

$(3) \Rightarrow(1)$ : Let $\rho$ be a good involution which is a quandle homomorphism. For any $x, y \in X, \rho\left(x^{y}\right)=\rho(x)^{\rho(y)}$. Since $\rho\left(x^{y}\right)=\rho(x)^{y}$ and $\rho(x)^{\rho(y)}=\rho(x)^{y^{-1}}$, we have $\rho(x)^{y}=\rho(x)^{y^{-1}}$.

$(1) \Rightarrow(4)$ : Let $\rho$ be a good involution. For any $x, y \in X, \rho\left(x^{y}\right)=\rho(x)^{y}=$ $\rho(x)^{y^{-1}}=\rho(x)^{\rho(y)}$. Therefore, $\rho$ is a quandle homomorphism. As seen above, the identity map is a good involution.

$(4) \Rightarrow(3)$ : It is obvious.

\section{The ASSOciated Group of A SYMmetric QuANDLE}

The associated group of a quandle $X$ is

$$
G_{X}=\left\langle x \in X ; x^{y}=y^{-1} x y \quad(x, y \in X)\right\rangle .
$$

The associated group with the natural map $\eta: X \rightarrow G_{X}$ has a universal mapping property; see 9, 13.

The associated group, $G_{(X, \rho)}$, of a symmetric quandle $(X, \rho)$ is defined by

$$
G_{(X, \rho)}=\left\langle x \in X ; x^{y}=y^{-1} x y \quad(x, y \in X), \quad \rho(x)=x^{-1} \quad(x \in X)\right\rangle .
$$

The natural map $\mu: X \rightarrow G_{(X, \rho)}$ is the composition of the inclusion map $X \rightarrow$ $F(X)$ and the projection map $F(X) \rightarrow G_{(X, \rho)}$, where $F(X)$ is the free group on $X$. The associated group $G_{(X, \rho)}$ with the natural map $\mu: X \rightarrow G_{(X, \rho)}$ has a universal mapping property as follows:

Proposition 4.1. Let $(X, \rho)$ be a symmetric quandle.

(1) The natural map $\mu: X \rightarrow G_{(X, \rho)}$ is a symmetric quandle homomorphism

$$
\mu:(X, \rho) \rightarrow\left(\operatorname{conj}\left(G_{(X, \rho)}\right), \operatorname{inv}\left(G_{(X, \rho)}\right)\right) .
$$

(2) Let $G$ be a group. Any symmetric quandle homomorphism $f:(X, \rho) \rightarrow$ $(\operatorname{conj}(G), \operatorname{inv}(G))$ "factors uniquely through $\mu$ "; that is, there exists a unique group homomorphism $f_{\sharp}: G_{(X, \rho)} \rightarrow G$ with $f=f_{\sharp} \circ \mu$, i.e., it makes the following diagram commutative:

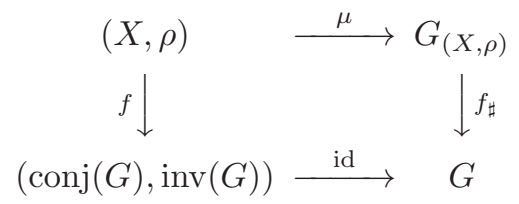

Proof. (1) For any $x, y \in X, x^{y}=y^{-1} x y$ and $\rho(x)=x^{-1}$ in $G_{(X, \rho)}$. This implies that $\mu\left(x^{y}\right)=\mu(x)^{\mu(y)}$ and $\mu \circ \rho(x)=\operatorname{inv}\left(G_{(X, \rho)}\right) \circ \mu(x)$.

(2) Let $f_{*}: F(X) \rightarrow G$ be the group homomorphism determined by the map $f: X \rightarrow G$. Since $f$ is a symmetric quandle homomorphism,

$$
f\left(x^{y}\right)=f(y)^{-1} f(x) f(y) \text { and } \quad f(\rho(x))=f(x)^{-1}
$$

for all $x, y \in X$. Thus $f_{*}: F(X) \rightarrow G$ induces a desired homomorphism $f_{\sharp}$ : $G_{(X, \rho)} \rightarrow G$. 
Proposition 4.1 is a symmetric quandle version of Proposition 2.1 of [9].

Example 4.2. (1) Let $(X, \rho)$ be the fundamental symmetric quandle of an unknot in $\mathbb{R}^{3}$ or an unknotted 2 -sphere in $\mathbb{R}^{4}$, i.e., $X=T_{2}=\left\{x_{1}, x_{2}\right\}$ and $\rho\left(x_{1}\right)=x_{2}$ (Example 2.5). The associated group $G_{X}$ of $X$ is $\mathbb{Z} \times \mathbb{Z}$, and the associated group $G_{(X, \rho)}$ is $\mathbb{Z}$.

(2) Let $(X, \rho)$ be the fundamental symmetric quandle of an unknotted projective plane in $\mathbb{R}^{4}$, i.e., $X=\{x\}$ and $\rho(x)=x$. The associated group $G_{X}$ is $\mathbb{Z}$, and the associated group $G_{(X, \rho)}$ is $\mathbb{Z} / 2 \mathbb{Z}$.

For a quandle $X$, an $X$-set is a set $Y$ equipped with a right action of the associated group $G_{X}$. For a symmetric quandle $(X, \rho)$, an $(X, \rho)$-set is a set $Y$ equipped with a right action of the associated group $G_{(X, \rho)}$. We denote by $y^{g}$ or by $y \cdot g$ the image of an element $y \in Y$ by the action $g \in G_{(X, \rho)}$. Then $y \cdot\left(x_{1} x_{2}\right)=\left(y \cdot x_{1}\right) \cdot x_{2}$, $y \cdot\left(x_{1}^{x_{2}}\right)=y \cdot\left(x_{2}^{-1} x_{1} x_{2}\right)$ and $y \cdot\left(\rho\left(x_{1}\right)\right)=y \cdot\left(x_{1}^{-1}\right)$ for $x_{1}, x_{2} \in X$ and $y \in Y$.

\section{Homology groups of A SYMmetric QuANDle}

Let $(X, \rho)$ be a symmetric quandle. Let $C_{n}=C_{n}(X)$ be the free abelian group generated by $n$-tuples $\left(x_{1}, \cdots, x_{n}\right)$ of elements of $X$ when $n$ is a positive integer, and let $C_{n}$ be $\{0\}$ otherwise. Define the boundary homomorphism $\partial_{n}: C_{n} \rightarrow C_{n-1}$ by

$\partial_{n}\left(x_{1}, \cdots, x_{n}\right)=\sum_{i=1}^{n}(-1)^{i}\left\{\left(x_{1}, \cdots, \widehat{x_{i}}, \cdots, x_{n}\right)-\left(x_{1}^{x_{i}}, \cdots, x_{i-1}^{x_{i}}, \widehat{x}_{i}, x_{i+1}, \cdots, x_{n}\right)\right\}$

for $n>1$ and $\partial_{n}=0$ for $n \leq 1$. Then $C_{*}=\left\{C_{n}, \partial_{n}\right\}$ is a chain complex (cf. [4, 5, 10, 11]).

Let $D_{n}^{\mathrm{Q}}$ be the subgroup of $C_{n}$ generated by the elements of

$$
\bigcup_{i=1}^{n-1}\left\{\left(x_{1}, \cdots, x_{n}\right) \in X^{n} \mid x_{i}=x_{i+1}\right\}
$$

and let $D_{n}^{\rho}$ be the subgroup of $C_{n}$ generated by the elements of

$$
\bigcup_{i=1}^{n}\left\{\left(x_{1}, \cdots, x_{n}\right)+\left(x_{1}^{x_{i}}, \cdots, x_{i-1}^{x_{i}}, \rho\left(x_{i}\right), x_{i+1}, \cdots, x_{n}\right) \mid x_{1}, \cdots, x_{n} \in X\right\} .
$$

Then $D_{*}^{\mathrm{Q}}=\left\{D_{n}^{\mathrm{Q}}, \partial_{n}\right\}$ and $D_{*}^{\rho}=\left\{D_{n}^{\rho}, \partial_{n}\right\}$ are subcomplexes of $C_{*}$. The former part of this fact is proved in [4 and the latter is seen as a special case of Lemma 5.1]

Define abelian groups $C_{n}^{\mathrm{R}}(X), C_{n}^{\mathrm{Q}}(X), C_{n}^{\mathrm{R}, \rho}(X)$ and $C_{n}^{\mathrm{Q}, \rho}(X)$ by

$$
\begin{array}{llll}
C_{n}^{\mathrm{R}}(X) & =C_{n}, & C_{n}^{\mathrm{Q}}(X) & =C_{n} / D_{n}^{\mathrm{Q}}, \\
C_{n}^{\mathrm{R}, \rho}(X) & =C_{n} / D_{n}^{\rho} \quad \text { and } \quad C_{n}^{\mathrm{Q}, \rho}(X)=C_{n} /\left(D_{n}^{\mathrm{Q}}+D_{n}^{\rho}\right),
\end{array}
$$

and we have chain complexes $C_{*}^{\mathrm{R}}(X), C_{*}^{\mathrm{Q}}(X), C_{*}^{\mathrm{R}, \rho}(X)$, and $C_{*}^{\mathrm{Q}, \rho}(X)$. Their homology groups are denoted by $H_{*}^{\mathrm{R}}(X), H_{*}^{\mathrm{Q}}(X), H_{*}^{\mathrm{R}, \rho}(X)$, and $H_{*}^{\mathrm{Q}, \rho}(X)$, and they are called the rack homology groups, quandle homology groups, symmetric rack homology groups, and symmetric quandle homology groups, respectively.

Let $Y$ be an $(X, \rho)$-set. Let $C_{n}(X)_{Y}$ be the free abelian group generated by the elements $\left(y, x_{1}, \ldots, x_{n}\right)$ where $y \in Y$ and $x_{1}, \ldots, x_{n} \in X$ when $n$ is a positive integer, let $C_{0}(X)_{Y}=\mathbb{Z}(Y)$, the free abelian group on $Y$, and let $C_{n}(X)_{Y}$ be $\{0\}$ for $n<0$. 
Define the boundary homomorphism $\partial_{n}: C_{n}(X)_{Y} \rightarrow C_{n-1}(X)_{Y}$ by

$$
\begin{aligned}
\partial_{n}\left(y, x_{1}, \cdots, x_{n}\right)=\sum_{i=1}^{n}(-1)^{i}\left\{\left(y, x_{1}, \cdots,\right.\right. & \left., x_{i}, \cdots, x_{n}\right) \\
& \left.-\left(y^{x_{i}}, x_{1}^{x_{i}}, \cdots, x_{i-1}^{x_{i}}, \widehat{x_{i}}, x_{i+1}, \cdots, x_{n}\right)\right\}
\end{aligned}
$$

for $n \geq 1$, and $\partial_{n}=0$ otherwise. Then $C_{*}(X)_{Y}=\left\{C_{n}(X)_{Y}, \partial_{n}\right\}$ is a chain complex (cf. [10, 11]).

Let $D_{n}^{\mathrm{Q}}(X)_{Y}$ be the subgroup of $C_{n}(X)_{Y}$ generated by the elements of

$$
\bigcup_{i=1}^{n-1}\left\{\left(y, x_{1}, \ldots, x_{n}\right) \mid x_{i}=x_{i+1}\right\}
$$

and let $D_{n}^{\rho}(X)_{Y}$ be the subgroup of $C_{n}(X)_{Y}$ generated by the elements of $\bigcup_{i=1}^{n}\left\{\left(y, x_{1}, \ldots, x_{n}\right)+\left(y^{x_{i}}, x_{1}^{x_{i}}, \ldots, x_{i-1}^{x_{i}}, \rho\left(x_{i}\right), x_{i+1}, \ldots, x_{n}\right) \mid y \in Y, x_{1}, \cdots, x_{n} \in X\right\}$.

Lemma 5.1. For each $n, \partial_{n}\left(D_{n}^{\mathrm{Q}}(X)_{Y}\right) \subset D_{n-1}^{\mathrm{Q}}(X)_{Y}$ and $\partial_{n}\left(D_{n}^{\rho}(X)_{Y}\right) \subset$ $D_{n-1}^{\rho}(X)_{Y}$. Hence $D_{*}^{\mathrm{Q}}(X)_{Y}=\left\{D_{n}^{\mathrm{Q}}(X)_{Y}, \partial_{n}\right\}$ and $D_{*}^{\rho}(X)_{Y}=\left\{D_{n}^{\rho}(X)_{Y}, \partial_{n}\right\}$ are subcomplexes of $C_{*}(X)_{Y}$.

Proof. It is seen by a direct calculation that $\partial_{n}\left(D_{n}^{\mathrm{Q}}(X)_{Y}\right) \subset D_{n-1}^{\mathrm{Q}}(X)_{Y}$ (cf. [4). We show that $\partial_{n}\left(D_{n}^{\rho}(X)_{Y}\right) \subset D_{n-1}^{\rho}(X)_{Y}$. Let $i \in\{1, \cdots, n\}$ be fixed. Recall that $\left(x^{z}\right)^{\left(y^{z}\right)}=\left(x^{y}\right)^{z}$ for $x \in X \cup Y$ and $y, z \in X$, and we denote $\left(x^{y}\right)^{z}$ by $x^{y z}$ for simplicity. In the following calculation, $\equiv$ stands for the congruence modulo $D_{n-1}^{\rho}(X)_{Y}$ :

$$
\begin{aligned}
& \partial_{n}\left(y^{x_{i}}, x_{1}^{x_{i}}, \cdots, x_{i-1}^{x_{i}}, \rho\left(x_{i}\right), x_{i+1}, \cdots, x_{n}\right) \\
& =\sum_{j=1}^{i-1}(-1)^{j}\left\{\left(y^{x_{i}}, x_{1}^{x_{i}}, \cdots, \widehat{x_{j}^{x_{i}}}, \cdots, x_{i-1}^{x_{i}}, \rho\left(x_{i}\right), x_{i+1}, \cdots, x_{n}\right)\right. \\
& \left.\quad-\left(y^{x_{j} x_{i}}, x_{1}^{x_{j} x_{i}}, \cdots, x_{j-1}^{x_{j} x_{i}}, \widehat{x_{j}}, x_{j+1}^{x_{i}}, \cdots, x_{i-1}^{x_{i}}, \rho\left(x_{i}\right), x_{i+1}, \cdots, x_{n}\right)\right\} \\
& \quad+(-1)^{i}\left\{\left(y^{x_{i}}, x_{1}^{x_{i}}, \cdots, x_{i-1}^{x_{i}}, x_{i+1}, \cdots, x_{n}\right)-\left(y, x_{1}, \cdots, x_{i-1}, x_{i+1}, \cdots, x_{n}\right)\right\} \\
& +\sum_{j=i+1}^{n}(-1)^{j}\left\{\left(y^{x_{i}}, x_{1}^{x_{i}}, \cdots, x_{i-1}^{x_{i}}, \rho\left(x_{i}\right), x_{i+1}, \cdots, \widehat{x_{j}}, \cdots, x_{n}\right)\right. \\
& \left.\quad-\left(y^{x_{i} x_{j}}, x_{1}^{x_{i} x_{j}}, \cdots, x_{i-1}^{x_{i} x_{j}}, \rho\left(x_{i}^{x_{j}}\right), x_{i+1}^{x_{j}}, \cdots, x_{j-1}^{x_{j}}, \widehat{x_{j}}, x_{j+1} \cdots, x_{n}\right)\right\} \\
& \equiv \sum_{j=1}^{i-1}(-1)^{j}\left\{-\left(y, x_{1}, \cdots, \widehat{x_{j}}, \cdots, x_{n}\right)+\left(y^{x_{j}}, x_{1}^{x_{j}}, \cdots, x_{j-1}^{x_{j}}, \widehat{x_{j}}, x_{j+1}, \cdots, x_{n}\right)\right\} \\
& \quad+(-1)^{i}\left\{\left(y^{x_{i}}, x_{1}^{x_{i}}, \cdots, x_{i-1}^{x_{i}}, x_{i+1}, \cdots, x_{n}\right)-\left(y, x_{1}, \cdots, x_{i-1}, x_{i+1}, \cdots, x_{n}\right)\right\} \\
& +\sum_{j=i+1}^{n}(-1)^{j}\left\{-\left(y, x_{1}, \cdots, \widehat{x_{j}}, \cdots, x_{n}\right)+\left(y^{x_{j}}, x_{1}^{x_{j}}, \cdots, x_{j-1}^{x_{j}}, \widehat{x_{j}}, x_{j+1}, \cdots, x_{n}\right)\right\} \\
& \equiv-\partial_{n}\left(y, x_{1}, \cdots, x_{n}\right) .
\end{aligned}
$$

Therefore, $\partial_{n}\left(D_{n}^{\rho}(X)_{Y}\right) \subset D_{n-1}^{\rho}(X)_{Y}$. 
Define $C_{n}^{\mathrm{R}}(X)_{Y}, C_{n}^{\mathrm{Q}}(X)_{Y}, C_{n}^{\mathrm{R}, \rho}(X)_{Y}$, and $C_{n}^{\mathrm{Q}, \rho}(X)_{Y}$ by

$$
\begin{aligned}
C_{n}^{\mathrm{R}}(X)_{Y} & =C_{n}(X)_{Y}, \\
C_{n}^{\mathrm{Q}}(X)_{Y} & =C_{n}(X)_{Y} / D_{n}^{\mathrm{Q}}(X)_{Y}, \\
C_{n}^{\mathrm{R}, \rho}(X)_{Y} & =C_{n}(X)_{Y} / D_{n}^{\rho}(X)_{Y}, \\
C_{n}^{\mathrm{Q}, \rho}(X)_{Y} & =C_{n}(X)_{Y} /\left(D_{n}^{\mathrm{Q}}(X)_{Y}+D_{n}^{\rho}(X)_{Y}\right),
\end{aligned}
$$

and we have chain complexes $C_{*}^{\mathrm{R}}(X)_{Y}, C_{*}^{\mathrm{Q}}(X)_{Y}, C_{*}^{\mathrm{R}, \rho}(X)_{Y}$ and $C_{*}^{\mathrm{Q}, \rho}(X)_{Y}$. The homology groups are denoted by $H_{*}^{\mathrm{R}}(X)_{Y}, H_{*}^{\mathrm{Q}}(X)_{Y}, H_{*}^{\mathrm{R}, \rho}(X)_{Y}$, and $H_{*}^{\mathrm{Q}, \rho}(X)_{Y}$, respectively.

For an abelian group $A$, we define the chain and cochain complexes $C_{*}^{W}(X, A)_{Y}=$ $C_{*}^{W}(X)_{Y} \otimes A$ and $C_{W}^{*}(X, A)_{Y}=\operatorname{Hom}\left(C_{*}^{W}(X)_{Y}, A\right)$, where $W=\mathrm{R}, \mathrm{Q},\{\mathrm{R}, \rho\}$ and $\{\mathrm{Q}, \rho\}$. The homology and cohomology groups are denoted by $H_{*}^{W}(X, A)_{Y}$ and $H_{W}^{*}(X, A)_{Y}$, respectively.

\section{QuANDLE COCYClE INVARIANTS $\Phi_{\theta}$ OF CLASSICAL LINKS}

Let $D$ be a diagram in $\mathbb{R}^{2}$ of an unoriented link in $\mathbb{R}^{3}$. Divide over-arcs at the crossings and call the arcs of the result semi-arcs of $D$. For example, the diagram $D$ in Figure 2 consists of 3 over-arcs and 6 semi-arcs.

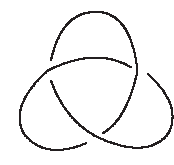

$D$

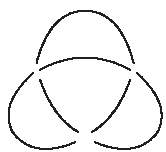

semi-arcs

Figure 2. semi-arcs

We say that an assignment of a normal orientation and an element of $X$ to each semi-arc of $D$ satisfies the coloring conditions if it satisfies the following two conditions (Figure 3):

- Suppose that two diagonal semi-arcs coming from an over-arc of $D$ at a crossing $v$ are labeled by $x_{1}$ and $x_{2}$. If the normal orientations are coherent, then $x_{1}=x_{2}$, otherwise $x_{1}=\rho\left(x_{2}\right)$.

- Suppose that two diagonal semi-arcs $e_{1}$ and $e_{2}$ which are under-arcs at a crossing $v$ are labeled by $x_{1}$ and $x_{2}$, and suppose that one of the semi-arcs coming from an over-arc of $D$ at $v$, say $e_{3}$, is labeled by $x_{3}$. We assume that the normal orientation of the over semi-arc $e_{3}$ points from $e_{1}$ to $e_{2}$. If the normal orientations of $e_{1}$ and $e_{2}$ are coherent, then $x_{1}^{x_{3}}=x_{2}$, otherwise $x_{1}^{x_{3}}=\rho\left(x_{2}\right)$. 

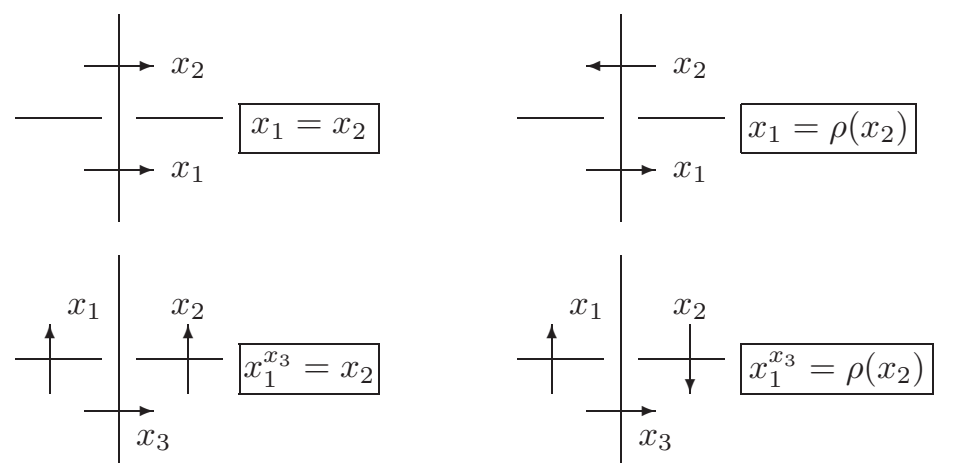

Figure 3. Coloring conditions

A basic inversion is an operation which reverses the normal orientation of a semi-arc and changes the element $x$ that is assigned the arc by $\rho(x)$. Note that the coloring conditions are preserved under basic inversions.

An $(X, \rho)$-coloring of $D$ is the equivalence class of an assignment of a normal orientation and an element of $X$ to each semi-arc of $D$ satisfying the coloring conditions. Here the equivalence relation is generated by basic inversions.

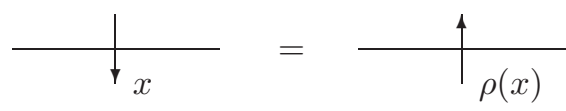

Figure 4. A basic inversion

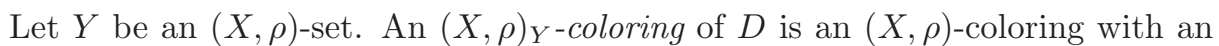
assignment of an element of $Y$ to each complementary region of $D$ satisfying the following condition (Figure 50):

- Suppose that two adjacent regions $f_{1}$ and $f_{2}$ which are separated by a semiarc, say $e$, are labeled by $y_{1}$ and $y_{2}$. Suppose that the semi-arc $e$ is labeled by $x$. If the normal orientation of $e$ points from $f_{1}$ to $f_{2}$, then $y_{1}^{x}=y_{2}$.

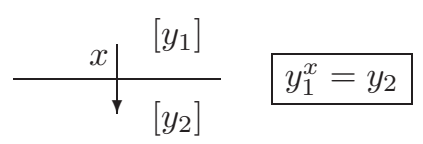

FiguRE 5. Coloring condition for regions

In figures, the label $y \in Y$ that is assigned a region is indicated by $[y]$. See Figure 5 .

Considering an $(X, \rho)$-set $Y$ consisting of a single element, we may regard an $(X, \rho)$-coloring as an $(X, \rho)_{Y}$-coloring.

Proposition 6.1. Let $(X, \rho)$ be a symmetric quandle, and let $Y$ be an $(X, \rho)$-set. If two link diagrams represent the same unoriented link type, then there is a bijection between the sets of $(X, \rho)$-colorings of the diagrams, and there is a bijection between the sets of $(X, \rho)_{Y}$-colorings. 
Proof. Suppose that $D$ and $D^{\prime}$ are diagrams related by a single Reidemeister move. Let $E$ be a 2-disk in $\mathbb{R}^{2}$ in which the Reidemeister move is applied. For each $(X, \rho)_{Y}$-coloring of $D$, its restriction to $D \backslash E\left(=D^{\prime} \backslash E\right)$ can be uniquely extended

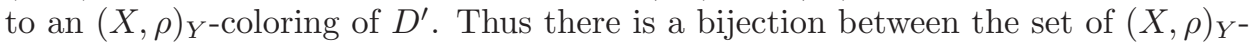
colorings of $D$ and that of $D^{\prime}$.

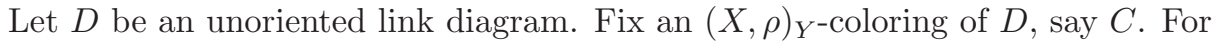
a crossing $v$ of $D$, there are four complementary regions of $D$ around $v$. (Some of them may be the same.) Choose one of them, say $f$, which we call a specified region for $v$, and let $y$ be the label of $f$. Let $e_{1}$ and $e_{2}$ be the under semi-arc and the over semi-arc at $v$, respectively, which face the region $f$. By basic inversions, we may assume that the normal orientations $n_{1}$ and $n_{2}$ of $e_{1}$ and $e_{2}$ point from $f$ to the opposite regions. Let $x_{1}$ and $x_{2}$ be the labels of them, respectively. The sign of $v$ with respect to the region $f$ is +1 (or -1 ) if the pair of normal orientations $\left(n_{2}, n_{1}\right)$ does (or does not) match the orientation of $\mathbb{R}^{2}$. The weight of $v$ is defined to be $\epsilon\left(y, x_{1}, x_{2}\right)$, where $\epsilon$ is the sign of $v$. See Figure 6 .

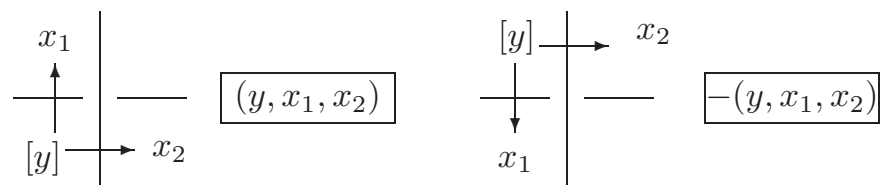

FiguRE 6. Weights

Lemma 6.2. As an element of $C_{2}^{\mathrm{R}, \rho}(X)_{Y}=C_{2}(X)_{Y} / D_{2}^{\rho}(X)_{Y}$, the weight of $v$ does not depend on the specified region.

Proof. When we change the specified region as in Figure 7, where the specified region is denoted by $*$, the weight $\left(y, x_{1}, x_{2}\right)$ changes to $-\left(y^{x_{2}}, x_{1}^{x_{2}}, \rho\left(x_{2}\right)\right),\left(y^{x_{1} x_{2}}\right.$, $\left.\rho\left(x_{1}\right)^{x_{2}}, \rho\left(x_{2}\right)\right)$ or $-\left(y^{x_{1}}, \rho\left(x_{1}\right), x_{2}\right)$. They are the same element of $C_{2}(X)_{Y} /$ $D_{2}^{\rho}(X)_{Y}$.

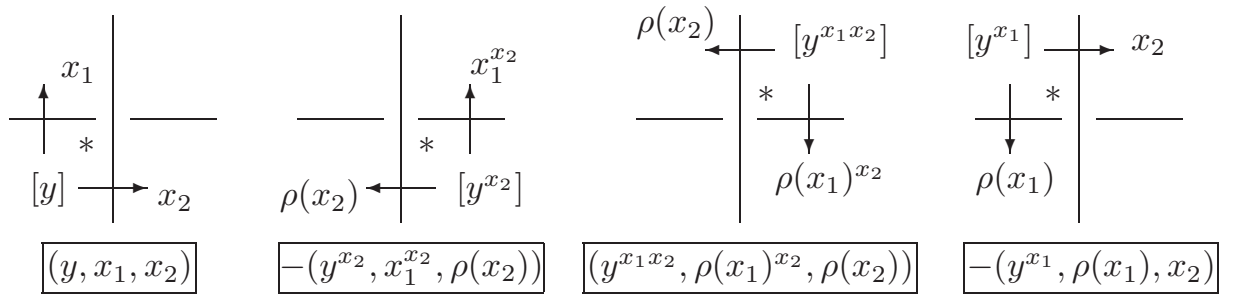

FiguRE 7 . Weights for the same crossing

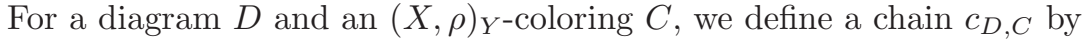

$$
c_{D, C}=\sum_{v} \epsilon\left(y, x_{1}, x_{2}\right) \in C_{2}^{\mathrm{R}, \rho}(X)_{Y} \text { or } C_{2}^{\mathrm{Q}, \rho}(X)_{Y},
$$

where $v$ runs over all crossings of $D$ and $\epsilon\left(y, x_{1}, x_{2}\right)$ is the weight of $v$. 


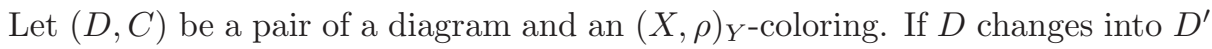
by a single Reidemeister move in a disk support $E$, then there is a unique $(X, \rho)_{Y^{-}}$ coloring, say $C^{\prime}$, of $D^{\prime}$ which is identical with $C$ outside $E$. In this situation, we say that $\left(D^{\prime}, C^{\prime}\right)$ is obtained from $(D, C)$ by a Reidemeister move (with support $E)$.

We say that $(D, C)$ and $\left(D^{\prime}, C^{\prime}\right)$ are Reidemeister move equivalent if they are related by a finite sequence of Reidemeister moves. The equivalence class of $(D, C)$ is called an $(X, \rho)_{Y}$-colored link.

Theorem 6.3. The homology class $\left[c_{D, C}\right] \in H_{2}^{\mathrm{Q}, \rho}(X)_{Y}$ is an invariant of an $(X, \rho)_{Y}$-colored link.

Proof. First we show that the chain $c_{D, C}$ is a 2-cycle of $C_{*}^{\mathrm{R}, \rho}(X)_{Y}$ and $C_{*}^{\mathrm{Q}, \rho}(X)_{Y}$. Let us fix a checkerboard coloring of the complementary regions of $D$. Using basic inversions, we assume that normal orientations of semi-arcs of $D$ point from the black regions to the white. Then we give an orientation to every semi-arc of $D$ such that the orientation vector followed by the normal orientation vector matches the orientation of $\mathbb{R}^{2}$. Assign a pair $(y, x) \in Y \times X$ to each semi-arc, where $y$ is the color of the black region and $x$ is the color of the semi-arc. See Figure 8 .

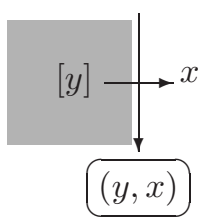

Figure 8. The label of a semi-arc

If the semi-arc is not a simple loop, then we give the initial point the weight $-(y, x)$ and the terminal point the weight $(y, x)$ with respect to the orientation of the semi-arc. For each crossing $v$ of $D$, we choose its specified region from the black regions. See Figure 9. If Figure 9(1) occurs at $v$, the weight of $v$ is $\left(y, x_{1}, x_{2}\right)$, and

$$
\begin{aligned}
\partial_{2}\left(y, x_{1}, x_{2}\right) & =-\left(y, x_{2}\right)+\left(y^{x_{1}}, x_{2}\right)+\left(y, x_{1}\right)-\left(y^{x_{2}}, x_{1}^{x_{2}}\right) \\
& \equiv-\left(y, x_{2}\right)-\left(y^{x_{1} x_{2}}, \rho\left(x_{2}\right)\right)+\left(y, x_{1}\right)+\left(y^{x_{1} x_{2}}, \rho\left(x_{1}\right)^{x_{2}}\right)
\end{aligned}
$$

$\bmod D_{1}^{\rho}(X)_{Y}$. The four terms are exactly the same with the weights assigned the endpoints of semi-arcs. See Figure 10, It is similar for Figure 9(2).

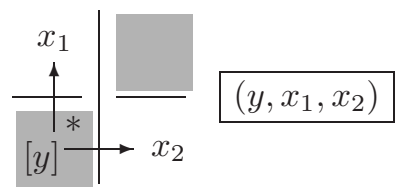

(1)

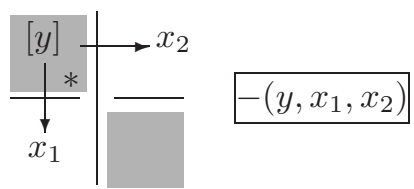

$(2)$

Figure 9. Weights 


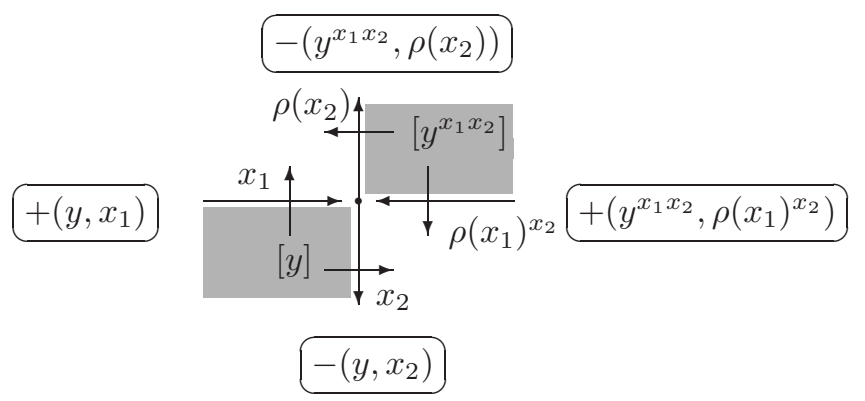

FiguRE 10. Weights

Since the weights of endpoints of semi-arcs are canceled, we see that $\partial_{2}\left(c_{D, C}\right) \equiv 0$ $\bmod D_{1}^{\rho}(X)_{Y}$. Therefore the chain $c_{D, C}$ is a 2-cycle of $C_{*}^{\mathrm{R}, \rho}(X)_{Y}$ and $C_{*}^{\mathrm{Q}, \rho}(X)_{Y}$.

It is sufficient to prove that if $(D, C)$ changes into $\left(D^{\prime}, C^{\prime}\right)$ by a single Reidemeister move, then $\left[c_{D, C}\right]=\left[c_{D^{\prime}, C^{\prime}}\right]$ in $H_{2}^{\mathrm{Q}, \rho}(X)_{Y}$. If the Reidemeister move is of type II, then $c_{D, C}$ equals $c_{D^{\prime}, C^{\prime}}$ in the chain group $C_{2}^{\mathrm{R}, \rho}(X)_{Y}$. If the Reidemeister move is of type III (Figure 11), then by choosing specified regions as in Figure 11] it is seen that the difference of $c_{D, C}$ and $c_{D^{\prime}, C^{\prime}}$ is $\pm \partial_{3}\left(y, x_{1}, x_{2}, x_{3}\right)$ for some $\left(y, x_{1}, x_{2}, x_{3}\right) \in Y \times X^{3}$; cf. [4, 10, 21, 22. In either case, $\left[c_{D, C}\right]=\left[c_{D^{\prime}, C^{\prime}}\right]$ in $H_{2}^{\mathrm{R}, \rho}(X)_{Y}$ and in $H_{2}^{\mathrm{Q}, \rho}(X)_{Y}$. If the Reidemeister move is of type $\mathrm{I}$, then the difference of $c_{D, C}$ and $c_{D^{\prime}, C^{\prime}}$ is $\pm(y, x, x)$ for some $(y, x) \in Y \times X$, which vanishes in $C_{2}^{\mathrm{Q}, \rho}(X)_{Y}$.
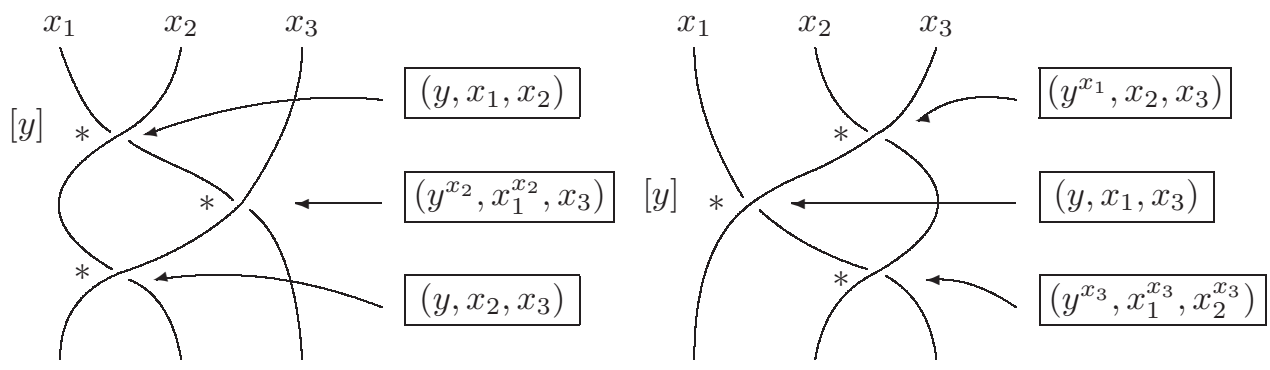

Figure 11. A Reidemeister move of type III

Let

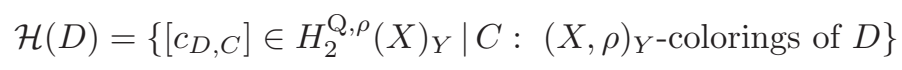

as a multi-set. (A multi-set means a set with (possible) repeats.)

For a 2-cocycle $\theta$ of the cochain complex $C_{\mathrm{Q}, \rho}^{*}(X, A)_{Y}$ with a coefficient group $A$, let

$$
\Phi_{\theta}(D)=\left\{\theta\left(c_{D, C}\right) \in A \mid C:(X, \rho)_{Y} \text {-colorings of } D\right\}
$$

as a multi-set.

Corollary 6.4. The multi-sets $\mathcal{H}(D)$ and $\Phi_{\theta}(D)$ are invariants of the link type of D. 
By definition of $C_{\mathrm{Q}, \rho}^{*}(X, A)_{Y}$, the linear extension $\theta: \mathbb{Z}\left(Y \times X^{2}\right) \rightarrow A$ of a map $\theta: Y \times X^{2} \rightarrow A$ is a 2-cocycle of $C_{\mathrm{Q}, \rho}^{*}(X, A)_{Y}$ if and only if the following conditions (1)-(3) are satisfied:

(1) for any $\left(y, x_{1}, x_{2}, x_{3}\right) \in Y \times X^{3}$,

$$
\begin{aligned}
-\theta\left(y, x_{2}, x_{3}\right)+\theta\left(y^{x_{1}}, x_{2}, x_{3}\right)+\theta\left(y, x_{1}, x_{3}\right) & \\
& -\theta\left(y^{x_{2}}, x_{1}^{x_{2}}, x_{3}\right)-\theta\left(y, x_{1}, x_{2}\right)+\theta\left(y^{x_{3}}, x_{1}^{x_{3}}, x_{2}^{x_{3}}\right)=0,
\end{aligned}
$$

(2) for any $(y, x) \in Y \times X, \theta(y, x, x)=0$, and

(3) for any $\left(y, x_{1}, x_{2}\right) \in Y \times X^{2}$,

$$
\theta\left(y, x_{1}, x_{2}\right)+\theta\left(y^{x_{1}}, \rho\left(x_{1}\right), x_{2}\right)=0 \quad \text { and } \quad \theta\left(y, x_{1}, x_{2}\right)+\theta\left(y^{x_{2}}, x_{1}^{x_{2}}, \rho\left(x_{2}\right)\right)=0 .
$$

We call these conditions the symmetric quandle 2-cocycle conditions.

Example 6.5. Let $X$ be the order 4 trivial quandle $T_{4}=\left\{e_{1}, e_{1}^{\prime}, e_{2}, e_{2}^{\prime}\right\}$, and let $\rho: X \rightarrow X$ be a good involution with $\rho\left(e_{i}\right)=e_{i}^{\prime}(i=1,2)$ (see Proposition 3.1). Let $Y=\{e\}$, which is an $(X, \rho)$-set. Define a map $\theta: Y \times X^{2} \rightarrow \mathbb{Z}$ by

$$
\theta=\chi_{\left(e, e_{1}, e_{2}\right)}+\chi_{\left(e, e_{1}^{\prime}, e_{2}^{\prime}\right)}-\chi_{\left(e, e_{1}^{\prime}, e_{2}\right)}-\chi_{\left(e, e_{1}, e_{2}^{\prime}\right)},
$$

where $\chi_{(e, a, b)}$ is defined by $\chi_{(e, a, b)}(e, x, y)=1$ if $(e, x, y)=(e, a, b)$ and $\chi_{(e, a, b)}(e, x, y)$ $=0$ otherwise. Then $\theta$ satisfies the symmetric quandle 2-cocycle conditions, and the linear extension $\theta: \mathbb{Z}\left(Y \times X^{2}\right) \rightarrow \mathbb{Z}$ is a 2 -cocycle.

Let $D$ be a diagram of an unoriented $(2, m)$-torus link with $2 m$ crossings. There

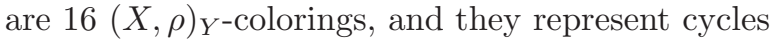

$$
m(e, a, b)+m(e, b, a)
$$

for $a, b \in X$. When we evaluate these cycles by $\theta$, we have

$$
\theta(m(e, a, b)+m(e, b, a))= \begin{cases}m & \text { if }(a, b)=\left(e_{i}, e_{j}\right),\left(e_{i}^{\prime}, e_{j}^{\prime}\right) \text { for } i \neq j, \\ -m & \text { if }(a, b)=\left(e_{i}^{\prime}, e_{j}\right),\left(e_{i}, e_{j}^{\prime}\right) \text { for } i \neq j, \\ 0 & \text { otherwise }\end{cases}
$$

Thus $\Phi_{f}(D)=\{m, m, m, m,-m,-m,-m,-m, 0, \cdots, 0(8$ times $)\}$.

Proposition 6.6. Let $(X, \rho), Y$, and $f$ be as in Example 6.5. Let $D$ be a diagram of a 2-component unoriented link $L$. Then $\Phi_{\theta}(D)=\{m, m, m, m,-m,-m,-m,-m$, $0, \cdots, 0(8$ times $)\}$, where $m$ is the linking number of $D$ when we give an orientation to $D$.

Proof. Give an orientation to $D=D_{1} \cup D_{2}$, and denote this oriented diagram by $D^{+}$. The semi-arcs of $D$ can be assigned a normal orientation such that the orientation vector of $D^{+}$followed by the normal orientation vector matches the orientation of $\mathbb{R}^{2}$. For each $(X, \rho)_{Y}$-coloring, we take a representative such that each semi-arc has a normal vector this way. Then each semi-arc is labeled by an element of $X$. The complementary regions are always colored by $e \in Y$. (This coloring is the $X_{Y}$-coloring of an oriented link diagram in the sense of [4, 21, etc.) Since $X$ is a trivial quandle, the labels of semi-arcs of the same knot component $D_{k}(k=1,2)$ are the same. Let $x_{k}$ be the label of $D_{k}$. There are 16 admissible colorings according to $\left(x_{1}, x_{2}\right) \in X \times X$. The linking number $\operatorname{Lk}\left(D^{+}\right)$of $D^{+}$is the sum of signs of crossings where $D_{i}$ is lower and $D_{j}$ is upper for $i \neq j$. When $\left(x_{1}, x_{2}\right)=\left(e_{i}, e_{j}\right)$ or $\left(e_{i}^{\prime}, e_{j}^{\prime}\right)$ with $i \neq j$, then the value $\theta(e, a, b)$ at a crossing $v$ is the sign of $v$ if $D_{i}$ is lower and $D_{j}$ is upper, or 0 otherwise. Thus for these colorings $C, \theta\left(c_{D, C}\right)=\operatorname{Lk}\left(D^{+}\right)$. When $\left(x_{1}, x_{2}\right)=\left(e_{i}^{\prime}, e_{j}\right)$ or $\left(e_{i}, e_{j}^{\prime}\right)$ with $i \neq j$, then the 
value $\theta(e, a, b)$ at a crossing $v$ is the negative of the sign of $v$ if $D_{i}$ is lower and $D_{j}$ is upper, or 0 otherwise. Thus for these colorings $C, \theta\left(c_{D, C}\right)=-\operatorname{Lk}\left(D^{+}\right)$. When $\left(x_{1}, x_{2}\right)$ is not $\left( \pm e_{i}, \pm e_{j}\right)$ with $i \neq j$, then the value $\theta(e, a, b)$ at a crossing $v$ is 0 . Thus for such a coloring $C, \theta\left(c_{D, C}\right)=0$.

The invariants $\mathcal{H}(D)$ and $\Phi_{\theta}(D)$ are analogies of invariants of oriented links in the sense of [4, 6, 21, which we denote by $\mathcal{H}^{\text {ori }}$ and $\Phi_{\theta}^{\text {ori }}$. We now recall them. Let $D^{+}$be an oriented diagram such that $D$ is obtained from $D^{+}$by forgetting the orientation. Using the orientation, we assign the semi-arcs of $D^{+}$normal orientations such that the orientation vector followed by the normal orientation vector matches the orientation of $\mathbb{R}^{2}$. An $X_{Y}$-coloring of $D^{+}$is an assignment of an element of $X$ to each semi-arc and an element of $Y$ to each complementary region of $D^{+}$satisfying the conditions illustrated on the left of Figure 3 and in Figure 5 . The weight of each crossing is defined as in Figure 6. Let $c_{D^{+}, C}$ be the sum of the weights of all crossings of $D$. It is a 2-cycle of $C_{*}^{\mathrm{Q}}(X)_{Y}$, and the homology class $\left[c_{D^{+}, C}\right] \in H_{2}^{\mathrm{Q}}(X)_{Y}$ is a colored link invariant [4, 6, 21]. Let $\mathcal{H}^{\text {ori }}\left(D^{+}\right)=\left\{\left[c_{D^{+}, C}\right] \in H_{2}^{\mathrm{Q}}(X)_{Y} \mid C\right.$ : $X_{Y^{-}}$colorings of $\left.D^{+}\right\}$and $\Phi_{\theta}^{\text {ori }}\left(D^{+}\right)=\left\{\theta\left(c_{D^{+}, C}\right) \in A \mid C: X_{Y^{-}}\right.$-colorings of $\left.D^{+}\right\}$as multi-sets, where $\theta$ is a 2-cocycle of $C_{\mathrm{Q}}^{*}(X, A)_{Y}$. Then $\mathcal{H}^{\text {ori }}\left(D^{+}\right)$and $\Phi_{\theta}^{\text {ori }}\left(D^{+}\right)$are oriented link invariants.

Note that if $\theta: \mathbb{Z}\left(Y \times X^{2}\right) \rightarrow A$ is a 2-cocycle of $C_{\mathrm{Q}, \rho}^{*}(X, A)_{Y}$, then $\theta$ is a 2-cocycle of $C_{\mathrm{Q}}^{*}(X, A)_{Y}$.

Theorem 6.7. Let $(X, \rho)$ be a symmetric quandle, $Y$ an $(X, \rho)$-set, $A$ an abelian group, and $\theta$ a 2-cocycle of $C_{\mathrm{Q}, \rho}^{*}(X, A)_{Y}$. Let $D$ be a diagram of an unoriented link in $\mathbb{R}^{3}$. Let $D^{+}$be an arbitrarily oriented diagram of $D$. Then

$$
\Phi_{\theta}(D)=\Phi_{\theta}^{\mathrm{ori}}\left(D^{+}\right) .
$$

Proof. Assign a normal orientation to each semi-arc of $D$ (and of $D^{+}$) such that the orientation vector determined from $D^{+}$followed by the normal orientation vector matches the orientation of $\mathbb{R}^{2}$. For each $(X, \rho)_{Y}$-coloring $C$ of $D$, we take a representative of $C$ such that the normal orientation of each semi-arc is the same with the normal orientation. Then $C$ determines an assignment of an element of $X$ to each semi-arc. Let $C^{+}$be this assignment, together with the same assignment of an element of $Y$ to each complementary region as $C$. Then $C^{+}$is an $X_{Y}$-coloring for the oriented diagram $D^{+}$in the sense of [4, 21]. Thus there is a bijection

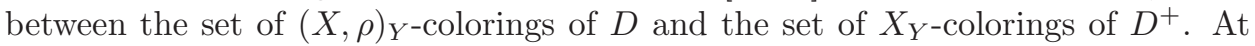
each crossing, the weights for $(D, C)$ and $\left(D^{+}, C^{+}\right)$evaluated by $\theta$ are the same, and hence we have

$$
\theta\left(c_{D, C}\right)=\theta\left(c_{D^{+}, C^{+}}\right)
$$

and $\Phi_{\theta}\left(D^{+}\right)=\Phi_{\theta}^{\text {ori }}(D)$.

Corollary 6.8. Let $(X, \rho)$ be a symmetric quandle, $Y$ an $(X, \rho)$-set, $A$ an abelian group, and $\theta$ a 2-cocycle of $C_{\mathrm{Q}}^{*}(X, A)_{Y}$. The invariant $\Phi_{\theta}^{\text {ori }}$ does not depend on the orientation of a link if $\theta$ is cohomologous in $C_{*}^{\mathrm{Q}}(X, A)_{Y}$ to a 2-cocycle satisfying the symmetric quandle 2-cocycle conditions. 


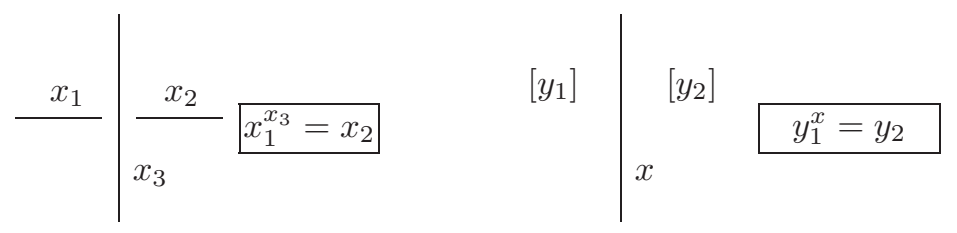

Figure 12. Coloring conditions

\section{A QUiCK CALCUlation OF $\Phi_{\theta}$}

Throughout this section, we assume that $X$ is a kei and $\rho$ is the identity map of $X$ (cf. Proposition 3.4). Under this assumption, the definition of the invariants $\Phi_{\theta}$ can be greatly simplified.

Let $D$ be a diagram in $\mathbb{R}^{2}$ of an unoriented link in $\mathbb{R}^{3}$. An $X$-coloring of $D$ is an assignment of an element of $X$ to each arc of $D$ such that for each crossing of $D$, $x_{1}^{x_{3}}=x_{2}$ holds, where $x_{1}$ and $x_{2}$ are elements of $X$ assigned the under-arcs and $x_{3}$ is the element assigned the over-arc as in Figure 12.

Let $Y$ be an $X$-set such that $y^{x}=y^{x^{-1}}$ for any $(x, y) \in X \times Y$. An $X_{Y^{-}}$ coloring of $D$ is an $X$-coloring of $D$ with an assignment of an element of $Y$ to each complementary region of $D$ such that, for each arc of $D, y_{1}^{x}=y_{2}$ holds, where $y_{1}$ and $y_{2}$ are elements of $Y$ assigned the regions separated by the arc, and $x$ is the element assigned the arc as in Figure 12

Since $X$ is a kei and $\rho=\operatorname{id}_{X}$, we see the following:

(1) An $X$-set $Y$ with $y^{x}=y^{x^{-1}}$ for any $(x, y) \in X \times Y$ is an $(X, \rho)$-set, and vice versa.

(2) Forgetting normal orientations, we have a bijection from the set of $(X, \rho)$ colorings of $D$ to the set of $X$-colorings of $D$.

(3) Forgetting normal orientations, we have a bijection from the set of $(X, \rho)_{Y^{-}}$ colorings of $D$ to the set of $X_{Y}$-colorings of $D$.

The following is well known, which is a special case of Proposition 6.1

Proposition 7.1 (cf. 4, 9, 13, 18, 22]). Let $X$ be a kei and $Y$ be an $X$-set such that $y^{x}=y^{x^{-1}}$ for any $(x, y) \in X \times Y$. If two link diagrams represent the same unoriented link type, then there is a bijection between the sets of $X$-colorings of the diagrams, and there is a bijection between the sets of $X_{Y}$-colorings.

Let $D$ be an unoriented link diagram. Fix an $X_{Y^{-}}$-coloring of $D$, say $C$. For a crossing $v$ of $D$, there are four complementary regions of $D$ around $v$. Choose one of them, and let $y$ be the element of $Y$ assigned the region. Let $x_{1}$ and $x_{2}$ be elements of $X$ assigned the under- and over-arcs facing the region as in Figure 13 , The weight of $v$ is $\epsilon\left(y, x_{1}, x_{2}\right)$, where $\epsilon$ is the sign of $v$. (Recall that the sign of a crossing of an unoriented link diagram is defined when a region around the crossing is specified.) 


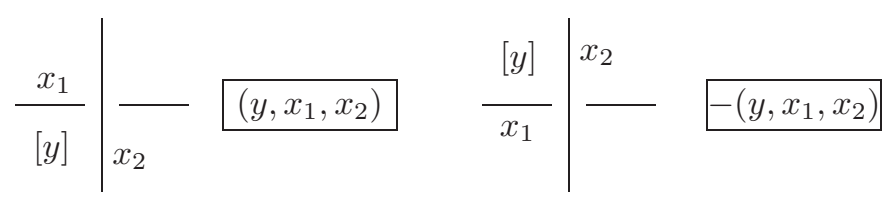

Figure 13. Weights

Now we obtain the chain $c_{D, C}$ as the sum of the weights of all crossings of $D$. The following is a special case of Theorem 6.3 where $X$ is a kei and $\rho$ is the identity map.

Theorem 7.2. The homology class $\left[c_{D, C}\right] \in H_{2}^{\mathrm{Q}, \rho}(X)_{Y}$ is an invariant of an $X_{Y^{-}}$colored link.

Let $\mathcal{H}(D)=\left\{\left[c_{D, C}\right] \in H_{2}^{\mathrm{Q}, \rho}(X)_{Y} \mid C: X_{Y}\right.$-colorings of $\left.D\right\}$ and $\Phi_{\theta}(D)=\left\{\theta\left(c_{D, C}\right)\right.$ $\in A \mid C: X_{Y^{-}}$-colorings of $\left.D\right\}$ as multi-sets, where $\theta$ is a 2-cocycle of the cochain complex $C_{\mathrm{Q}, \rho}^{*}(X, A)_{Y}$.

Corollary 7.3. The multi-sets $\mathcal{H}(D)$ and $\Phi_{\theta}(D)$ are invariants of the link type of D.

In order to obtain an invariant of $D$ as in Corollary 7.3 , one may restrict the range of colorings $C$ suitably in the definition of $\mathcal{H}(D)$ or $\Phi_{\theta}(D)$; for example, we have another invariant by assuming that $C$ runs over all colorings of $D$ such that the unbounded region is colored by a specified element of $Y$ (cf. Example 7.4).

Example 7.4. Let $X$ be the dihedral quandle $R_{3}=\mathbb{Z} / 3 \mathbb{Z}$ of order 3 and $\rho$ be the identity map of $X$. Let $Y=X$ on which $X$ acts by the quandle operation. A map

$$
\theta: X \times X^{2} \rightarrow \mathbb{Z} / 3 \mathbb{Z} ; \theta(x, y, z)=(x-y)(y-z)^{2} z
$$

satisfies the symmetric quandle 2-cocycle conditions, and the linear extension $\theta$ : $\mathbb{Z}\left(X \times X^{2}\right) \rightarrow \mathbb{Z} / 3 \mathbb{Z}$ is a 2 -cocycle of the cochain complex $C_{\mathrm{Q}, \rho}^{*}(X, \mathbb{Z} / 3 \mathbb{Z})_{X}$. (It is a modified version of Mochizuki's cocycle [19.)

Thus, for an unoriented link diagram $D$ in $\mathbb{R}^{2}$, a multi-set

$$
\Phi_{\theta}(D)_{\infty=0}=\left\{\begin{array}{l|l}
\theta\left(c_{D, C}\right) \in \mathbb{Z} / 3 \mathbb{Z} & \begin{array}{l}
C: X_{X} \text {-colorings of } D \text { such that } \\
\text { the unbounded region is colored by } 0
\end{array}
\end{array}\right\}
$$

is an unoriented link invariant. For example, let $D$ be the diagram of a trefoil illustrated in Figure 14 Any coloring $C$ of $D$ such that the unbounded region is 0 is given as in Figure 14, where $a, b \in X=\mathbb{Z} / 3 \mathbb{Z}$, and the 2-chain derived from the colored diagram $(D, C)$ is

$$
c_{D, C}=(0, a, b)+(0, b,-a-b)+(0,-a-b, a) .
$$

Then we have

$$
\theta\left(c_{C, D}\right)= \begin{cases}0, & \text { if } a=b \\ 1, & \text { if } a \neq b\end{cases}
$$

and hence $\Phi_{\theta}(D)_{\infty=0}=\{0,0,0,1,1,1,1,1,1\}$, which is regarded as a multi-set with elements of $\mathbb{Z} / 3 \mathbb{Z}$. Let $D^{*}$ be the mirror image of $D$. Any coloring $C^{*}$ of $D^{*}$ such that the unbounded region is 0 is given as in Figure 15] and the 2-chain derived from the colored diagram $\left(D^{*}, C^{*}\right)$ is

$$
c_{D^{*}, C^{*}}=-(0, a, b)-(0, b,-a-b)-(0,-a-b, a)=-c_{D, C} .
$$




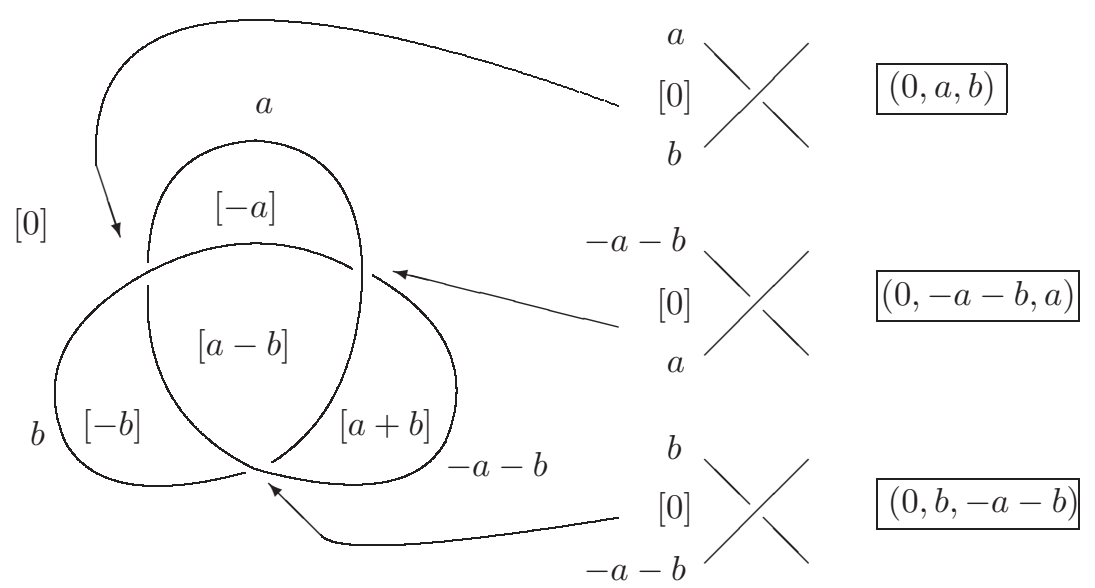

FiguRE 14. A diagram of a right-handed trefoil

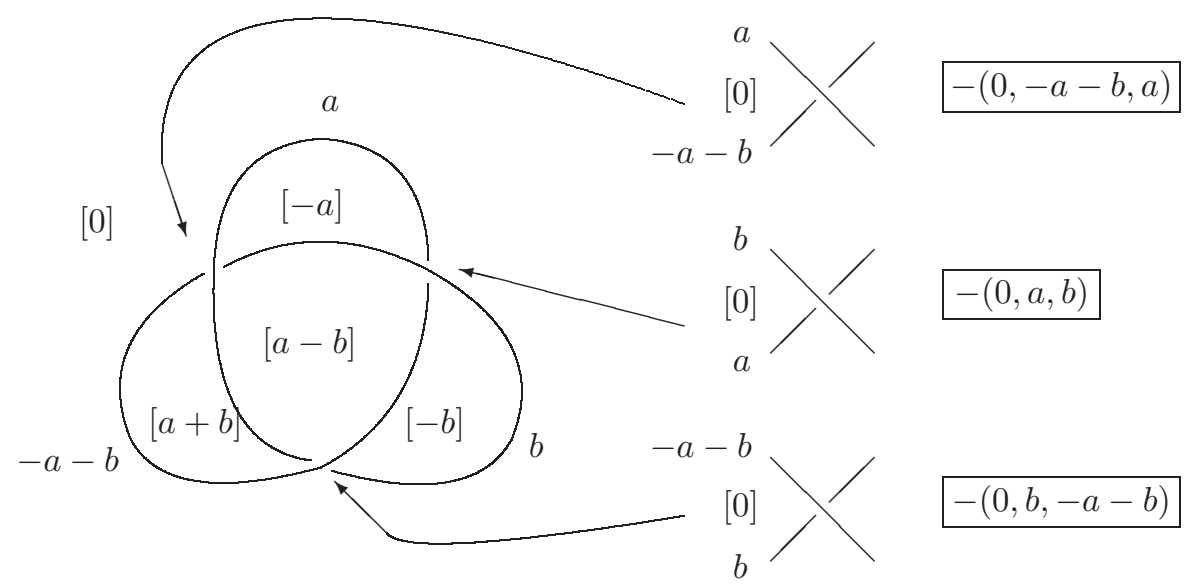

Figure 15. A diagram of a left-handed trefoil

Thus we have $\Phi_{\theta}\left(D^{*}\right)_{\infty=0}=\{0,0,0,-1,-1,-1,-1,-1,-1\}$. As a consequence, we see that the trefoil is chiral. This argument is an unoriented link version of the argument given by Rourke and Sanderson [21, 22.

As seen in Example 7.4 we can calculate the quandle cocycle invariant $\Phi_{\theta}^{\text {ori }}$ by calculating $\Phi_{\theta}$ without considering the sign and the orientation of arcs at each crossing if $X$ is a kei and $\rho$ is the identity map. This gives a practical method of calculation of some $\Phi_{\theta}^{\text {ori }}$; cf. [12, 25].

\section{Quandle COCYCle inVARIANTS OF SURFACE-LinKS}

Let $D$ be a diagram in $\mathbb{R}^{3}$ of an unoriented surface-link in $\mathbb{R}^{4}$. Divide oversheets at the double curves; we call the sheets of the result semi-sheets of $D$. Each semi-sheet is a compact orientable surface in $\mathbb{R}^{3}$ (cf. [14]).

We say that an assignment of a normal orientation and an element of $X$ to each semi-sheet of $D$ satisfies the coloring conditions if it satisfies the following conditions (Figure 16). 
- Suppose that two diagonal semi-sheets coming from an over-sheet of $D$ about a double curve are labeled by $x_{1}$ and $x_{2}$. If the normal orientations are coherent, then $x_{1}=x_{2}$; otherwise $x_{1}=\rho\left(x_{2}\right)$.

- Suppose that two diagonal semi-sheets $e_{1}$ and $e_{2}$ which are under-sheets about a double curve are labeled by $x_{1}$ and $x_{2}$, and suppose that one of the two semi-sheets coming from an over-sheet of $D$, say $e_{3}$, is labeled by $x_{3}$. We assume that the normal orientation of the over semi-arc $e_{3}$ points from $e_{1}$ to $e_{2}$. If the normal orientations of $e_{1}$ and $e_{2}$ are coherent, then $x_{1}^{x_{3}}=x_{2}$; otherwise $x_{1}^{x_{3}}=\rho\left(x_{2}\right)$.
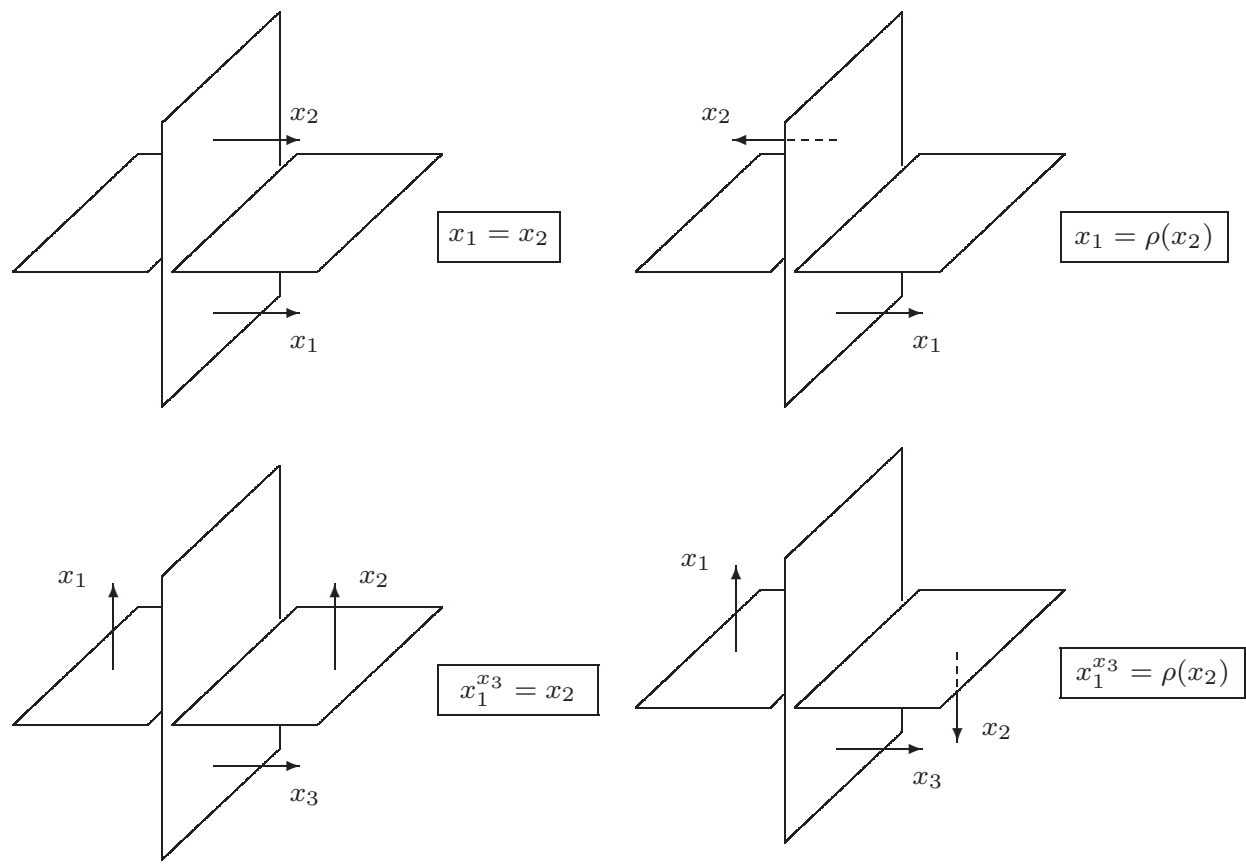

Figure 16. Coloring conditions

A basic inversion is an operation which reverses the normal orientation of a semi-sheet and changes the element $x$ assigned the sheet by $\rho(x)$. See Figure 17 The coloring conditions are preserved under basis inversions.

An $(X, \rho)$-coloring of $D$ is the equivalence class of an assignment of a normal orientation and an element of $X$ to each semi-sheet of $D$ satisfying the coloring conditions. Here the equivalence relation is generated by basic inversions. 

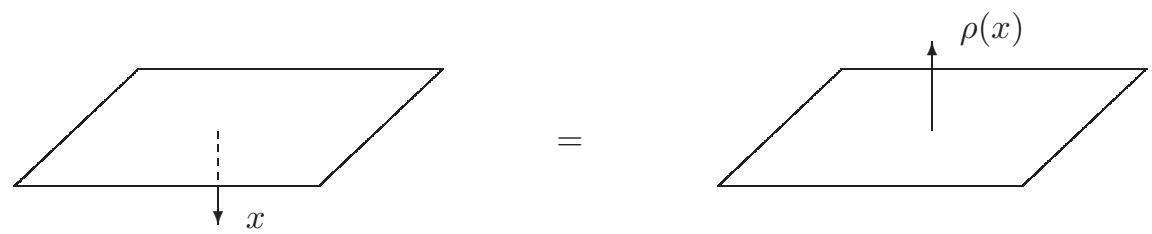

FiguRe 17. A basic inversion

Let $Y$ be an $(X, \rho)$-set. An $(X, \rho)_{Y}$-coloring of $D$ is an $(X, \rho)$-coloring with an assignment of an element of $Y$ to each complementary region of $D$ satisfying the following condition (Figure 18):

- Suppose that two adjacent regions $f_{1}$ and $f_{2}$ which are separated by a semi-sheet, say $e$, are labeled by $y_{1}$ and $y_{2}$. Suppose that the semi-sheet $e$ is labeled by $x$. If the normal orientation of $e$ points from $f_{1}$ to $f_{2}$, then $y_{1}^{x}=y_{2}$.

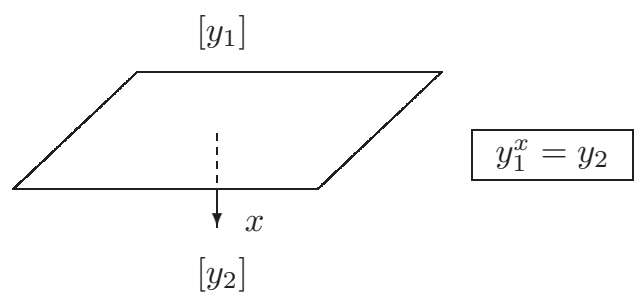

Figure 18. Coloring condition for regions

In figures, the label $y \in Y$ assigned a region is indicated by $[y]$. See Figure 18 ,

Proposition 8.1. Let $(X, \rho)$ be a symmetric quandle and $Y$ be an $(X, \rho)$-set. If two diagrams represent the same unoriented surface-link type, then there is a bijection between the sets of $(X, \rho)$-colorings of the diagrams and a bijection between the sets of $(X, \rho)_{Y}$-colorings.

Proof. Suppose that $D$ and $D^{\prime}$ are diagrams related by a single Roseman move [8, 20. Let $E$ be a 3 -disk in $\mathbb{R}^{3}$ in which the Roseman move is applied. For each $(X, \rho)_{Y}$-coloring of $D$, its restriction to $D \backslash E\left(=D^{\prime} \backslash E\right)$ can be uniquely extended

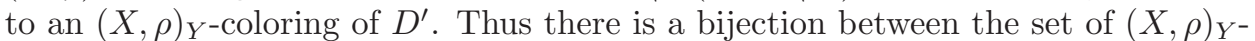
colorings of $D$ and that of $D^{\prime}$.

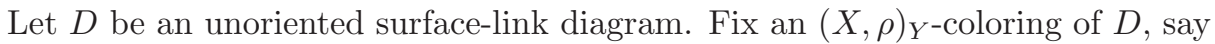
$C$. For a triple point $v$ of $D$, there are eight complementary regions of $D$ around $v$. (Some of them may be the same.) Choose one of them, say $f$, which we call $a$ specified region for $v$, and let $y$ be the label of $f$.

Let $e_{1}, e_{2}$, and $e_{3}$ be the lower semi-sheet, the middle semi-sheet, and the upper semi-sheet at $v$, respectively, which face the region $f$. By basic inversions, we assume that the normal orientations $n_{1}, n_{2}$ and $n_{3}$ of them point from $f$ to the opposite regions. Let $x_{1}, x_{2}$ and $x_{3}$ be the labels of them, respectively. The sign of $v$ with respect to the region $f$ is +1 (or -1 ) if the pair of normal orientations 
$\left(n_{3}, n_{2}, n_{1}\right)$ does (or does not) match the orientation of $\mathbb{R}^{3}$. The weight of $v$ is defined to be $\epsilon\left(y, x_{1}, x_{2}, x_{3}\right)$, where $\epsilon$ is the sign of $v$. See Figure 19 ,
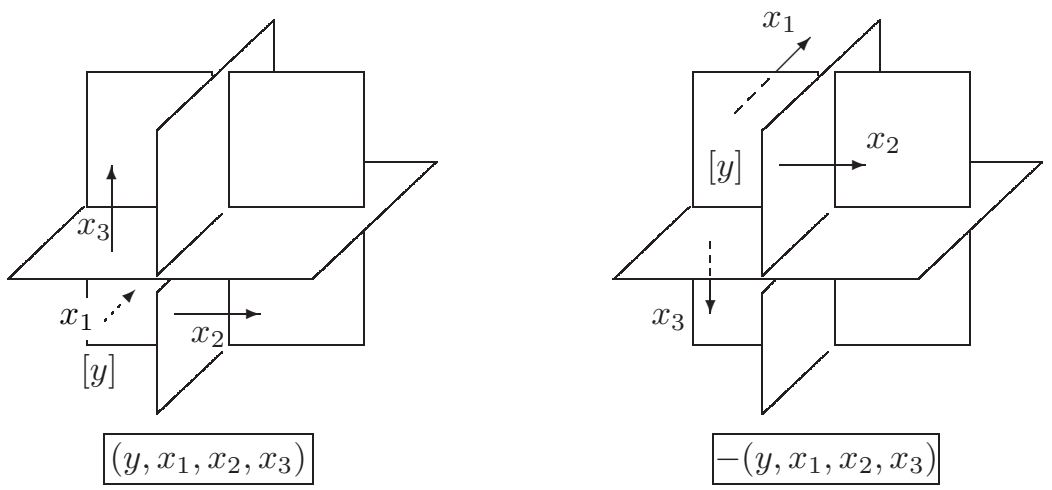

FiguRE 19. Weights

Lemma 8.2. As an element of $C_{3}^{\mathrm{R}, \rho}(X)_{Y}=C_{3}(X)_{Y} / D_{3}^{\rho}(X)_{Y}$, the weight of $v$ does not depend on the specified region.

Proof. When we change the specified region, the difference of the weights belongs to $D_{3}^{\rho}(X)_{Y}$.

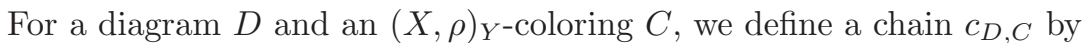

$$
c_{D, C}=\sum_{v} \epsilon\left(y, x_{1}, x_{2}, x_{3}\right) \in C_{3}^{\mathrm{R}, \rho}(X)_{Y} \text { or } C_{3}^{\mathrm{Q}, \rho}(X)_{Y},
$$

where $v$ runs over all triple points of $v$ and $\epsilon\left(y, x_{1}, x_{2}, x_{3}\right)$ is the weight of $v$.

Let $(D, C)$ be a pair of an unoriented surface-link diagram and an $(X, \rho)_{Y^{-}}$ coloring. If $D$ changes into $D^{\prime}$ by a single Roseman move in a 3-disk support $E$, then there is a unique $(X, \rho)_{Y}$-coloring, say $C^{\prime}$, of $D^{\prime}$ which is identical with $C$ outside $E$. In this situation, we say that $\left(D^{\prime}, C^{\prime}\right)$ is obtained from $(D, C)$ by a Roseman move (with support $E$ ).

We say that $(D, C)$ and $\left(D^{\prime}, C^{\prime}\right)$ are Roseman move equivalent if they are related by a finite sequence of Roseman moves. The equivalence class of $(D, C)$ is called an $(X, \rho)_{Y}$-colored surface-link.

Theorem 8.3. The chain $c_{D, C}$ is a 3-cycle of $C_{*}^{\mathrm{Q}, \rho}(X)_{Y}$, and the homology class $\left[c_{D, C}\right] \in H_{3}^{\mathrm{Q}, \rho}(X)_{Y}$ is an invariant of an $(X, \rho)_{Y}$-colored surface-link.

Proof. First we show that the chain $c_{D, C}$ is a 3 -cycle of $C_{*}^{\mathrm{Q}, \rho}(X)_{Y}$. Let us fix a checkerboard coloring of the complementary regions of $D$. Using basic inversions, we assume that normal orientations of semi-sheets of $D$ point from the black regions to the white. Give an orientation to each double curve of $D$ such that the orientation vector followed by the normal orientation vector of the over semi-sheet and the normal orientation vector of the under semi-sheet matches the orientation of $\mathbb{R}^{3}$. See Figure 20. 
For a double curve $\gamma$, choose a black region around $\gamma$, and let $y$ be the coloring assigned to this region. Let $x_{1}$ and $x_{2}$ be the color of the under semi-sheet and the color of the over semi-sheet around $\gamma$ which face the black region. Assign a triple $\left(y, x_{1}, x_{2}\right) \in Y \times X^{2}$ to the double curve $\gamma$. See Figure 20. It depends on a choice of the black region around $\gamma$, however it determines a unique element of $C_{*}^{\mathrm{Q}, \rho}(X)_{Y}$.

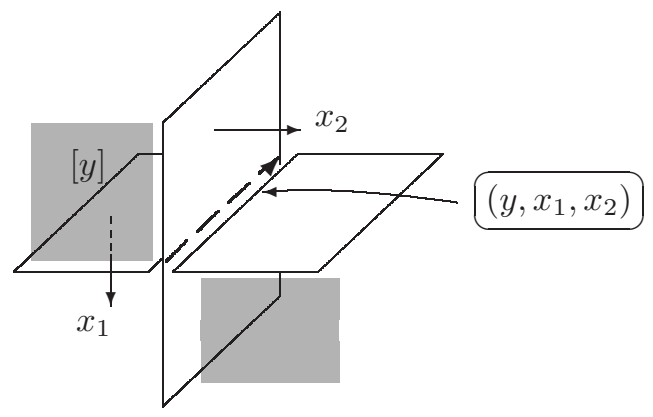

Figure 20. The label of a double point set

If the double curve is not a simple loop, then we give the initial point the weight $-\left(y, x_{1}, x_{2}\right)$ and the terminal point the weight $\left(y, x_{1}, x_{2}\right)$. For each triple point $v$ of $D$, we choose its specified region from the black regions. In the situation of Figure 21, the weight of $v$ is $\left(y, x_{1}, x_{2}, x_{3}\right)$, and

$$
\begin{aligned}
\partial_{3}\left(y, x_{1}, x_{2}, x_{3}\right)= & -\left(y, x_{2}, x_{3}\right)+\left(y^{x_{1}}, x_{2}, x_{3}\right)+\left(y, x_{1}, x_{3}\right)-\left(y^{x_{2}}, x_{1}^{x_{2}}, x_{3}\right) \\
& -\left(y, x_{1}, x_{2}\right)+\left(y^{x_{3}}, x_{1}^{x_{3}}, x_{2}^{x_{3}}\right) \\
\equiv & -\left(y, x_{2}, x_{3}\right)-\left(y^{x_{1} x_{3}}, x_{2}^{x_{3}}, \rho\left(x_{3}\right)\right)+\left(y, x_{1}, x_{3}\right) \\
& +\left(y^{x_{1} x_{2}}, \rho\left(x_{1}\right)^{x_{2}}, x_{3}\right)-\left(y, x_{1}, x_{2}\right)-\left(y^{x_{1} x_{3}}, \rho\left(x_{1}\right)^{x_{3}}, x_{2}^{x_{3}}\right),
\end{aligned}
$$

$\bmod D_{2}^{\rho}(X)_{Y}$. The six terms are exactly the same with the weights assigned the endpoints of double curves around $v$. Since the weights of endpoints of double curves connecting triple points must be canceled, we see that $\partial_{3}\left(c_{D, C}\right) \in C_{2}^{\mathrm{Q}, \rho}(X)_{Y}$ is the sum of the weights of initial or terminal points of the double curves, one of whose endpoints are triples points and the other whose endpoints are branch points. However, such weights are in the form $\pm(y, x, x)$ or $\pm(y, x, \rho(x))$ which vanish in $C_{2}^{\mathrm{Q}, \rho}(X)_{Y}$. Therefore the chain $c_{D, C}$ is a 3 -cycle of $C_{*}^{\mathrm{Q}, \rho}(X)_{Y}$. 

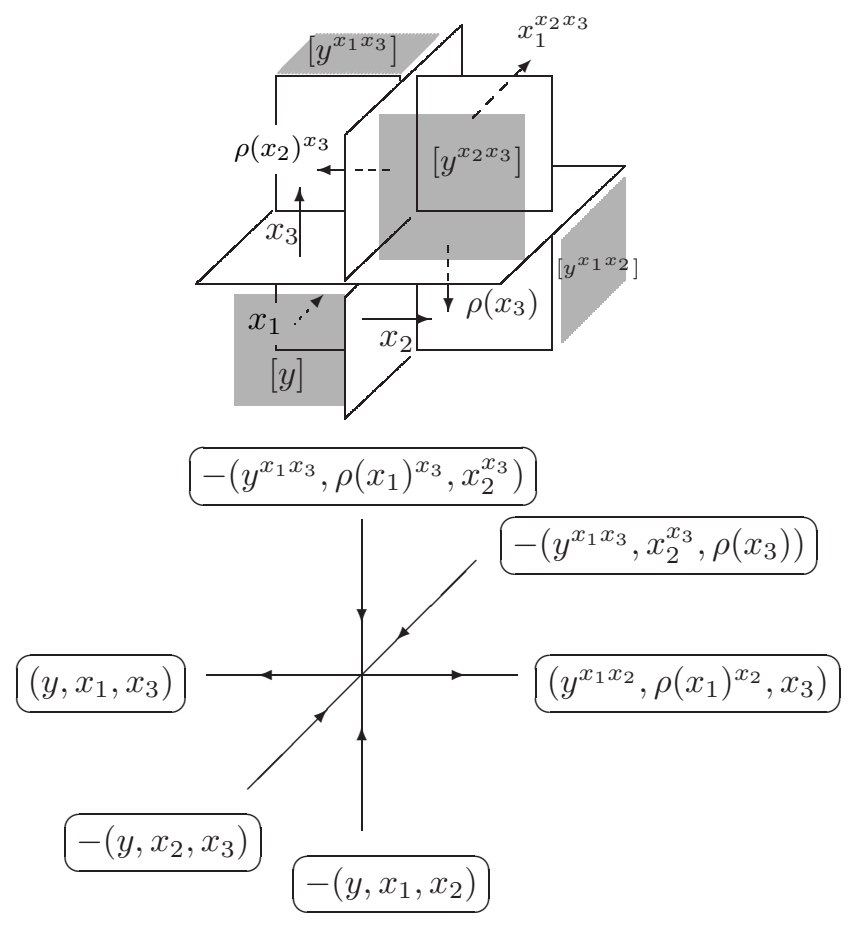

Figure 21. The label of a double curve

We show that if $(D, C)$ changes into $\left(D^{\prime}, C^{\prime}\right)$ by a Roseman move (whose support is a 3-disk $E)$, then $\left[c_{D, C}\right]=\left[c_{D^{\prime}, C^{\prime}}\right]$ in $H_{3}^{Q, \rho}(X)_{Y}$. The diagrams $D$ and $D^{\prime}$ and their colorings $C$ and $C^{\prime}$ are identical outside of $E$. The preimage of $E$ in the surface-link $F$ and $F^{\prime}$ described by $D$ and $D^{\prime}$ are disks in $F$ and $F^{\prime}$, respectively. Assign the disks orientations and project the orientation to the semi-sheets of $D$ and $D^{\prime}$ restricted in $E$. Assume that the normal orientation of semi-sheets which meets $E$ are compatible with these orientations with respect to the orientation of $\mathbb{R}^{3}$. For each triple point $v$ in $E$, there is a unique complementary region around $v$ such that the semi-sheets facing the region have normal orientations from the region. When we choose such a region as the specified region, the sum of weights of triple points of $D$ in $E$ and that of triple points of $D^{\prime}$ in $E$ differ by an element of $D_{3}^{\mathrm{Q}}(X)$ or $\partial\left(C_{4}^{\mathrm{Q}}(X)\right)$ by the same reason as in [4, 6]. Thus the difference of the weight sum for $(D, C)$ and for $\left(D^{\prime}, C^{\prime}\right)$ belongs to $D_{3}^{\mathrm{Q}}(X)+D_{3}^{\rho}(X)+\partial\left(C_{4}^{\mathrm{Q}}(X)\right)$.

Let

$$
\mathcal{H}(D)=\left\{\left[c_{D, C}\right] \in H_{3}^{\mathrm{Q}, \rho}(X)_{Y} \mid C:(X, \rho)_{Y \text {-colorings of } D\}}\right.
$$

as a multi-set. For a 3 -cocycle $\theta$ of the cochain complex $C_{\mathrm{Q}, \rho}^{*}(X, A)_{Y}$ with a coefficient group $A$, let

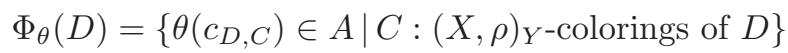

as a multi-set.

Corollary 8.4. The multi-sets $\mathcal{H}(D)$ and $\Phi_{\theta}(D)$ are invariants of the surface-link type of $D$. 
We recall quandle cocycle invariants of oriented surface-links in the sense of [4, 6, 21]. Let $D^{+}$be an oriented diagram such that $D$ is obtained from $D^{+}$by forgetting orientation. Using the orientation, we assign the semi-sheets of $D^{+}$normal orientations such that the orientation vectors followed by the normal orientation vector match the orientation of $\mathbb{R}^{3}$. An $X_{Y}$-coloring is an assignment of an element of $X$ to each semi-sheet and an element of $Y$ to each complementary region of $D$ satisfying the conditions illustrated on the left in Figure 16 and in Figure 18. The weight of each triple point is defined as in Figure 19. Let

$$
\mathcal{H}^{\text {ori }}\left(D^{+}\right)=\left\{\left[c_{D^{+}, C}\right] \in H_{3}^{\mathrm{Q}}(X)_{Y} \mid C: X_{Y^{-} \text {colorings of } D^{+}}\right\}
$$

and

$$
\Phi_{\theta}^{\text {ori }}\left(D^{+}\right)=\left\{\theta\left(c_{D^{+}, C}\right) \in A \mid C: X_{\left.Y^{-} \text {colorings of } D^{+}\right\}}\right.
$$

as multi-sets, where $\theta$ is a 3-cocycle of $C_{\mathrm{Q}}^{*}(X, A)_{Y}$.

If $\theta: \mathbb{Z}\left(Y \times X^{3}\right) \rightarrow A$ is a 3 -cocycle of $C_{\mathrm{Q}, \rho}^{*}(X, A)_{Y}$, then $\theta$ is a 3-cocycle of $C_{\mathrm{Q}}^{*}(X, A)_{Y}$.

Theorem 8.5. Let $(X, \rho)$ be a symmetric quandle, $Y$ an $(X, \rho)$-set, $A$ an abelian group, and $\theta$ a 3-cocycle of $C_{\mathrm{Q}, \rho}^{*}(X ; A)_{Y}$. Let $D$ be a diagram of an unoriented surface-link $F$ such that every component of $F$ is orientable. Let $D^{+}$be an arbitrarily oriented diagram of $D$. Then

$$
\Phi_{\theta}(D)=\Phi_{\theta}^{\text {ori }}\left(D^{+}\right) \text {. }
$$

Proof. Assign a normal orientation to each semi-sheet of $D$ (and $D^{+}$) such that the orientation vectors determined from $D^{+}$followed by the normal orientation vector match the orientation of $\mathbb{R}^{3}$. For each $(X, \rho)_{Y}$-coloring $C$ of $D$, take a representative of $C$ such that the normal orientation of each semi-sheet of $D$ is the same with the normal orientation. Then $C$ determines an assignment of an element of $X$ to each semi-sheet. Let $C^{+}$be this assignment, together with the same assignment of an element of $Y$ to each complementary region as $C$. Then $C^{+}$is an $X_{Y}$-coloring for the oriented diagram $D^{+}$in the sense of [4, 6, 21. Then there is a bijection between the set of $(X, \rho)_{Y}$-colorings of $D$ and the set of $X_{Y}$-colorings of $D^{+}$. At each triple point the weights for $(D, C)$ and $\left(D^{\prime}, C^{\prime}\right)$ evaluated by $\theta$ are the same, and we have

$$
\theta\left(c_{D, C}\right)=\theta\left(c_{D^{+}, C^{+}}\right)
$$

and $\Phi_{\theta}\left(D^{+}\right)=\Phi_{\theta}^{\text {ori }}(D)$.

Theorem 8.6. Let $(X, \rho)$ be a symmetric quandle, $Y$ an $(X, \rho)$-set and $\theta$ a 3 cocycle of $C_{\mathrm{Q}, \rho}^{*}(X, \mathbb{Z})_{Y}$. Suppose that for any $\left(y, x_{1}, x_{2}, x_{3}\right) \in Y \times X^{3}$,

$$
\left|\theta\left(\left(e, x_{i_{1}}, x_{i_{2}}, x_{i_{3}}\right)\right)\right| \leq 1 .
$$

Let $F$ be a surface-link and $D$ be a diagram of $F$ with an $(X, \rho)_{Y}$-coloring $C$. Then $\left|\theta\left(c_{D, C}\right)\right| \leq t(F)$, where $t(F)$ is the minimal triple point number of $F$.

Proof. Let $D^{\prime}$ be a diagram of $F$ such that the number of triple points is $t(F)$. Let

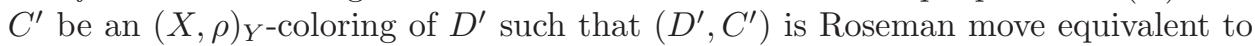
$(D, C)$. By Theorem 8.3, $\theta\left(c_{D, C}\right)=\theta\left(c_{D^{\prime}, C^{\prime}}\right)$. By definition, the chain $c\left(D^{\prime}, C^{\prime}\right)$ is expressed as $\sum_{i=1}^{t(F)} \epsilon_{i}\left(y_{i}, x_{i_{1}} x_{i_{2}}, x_{i_{3}}\right)$ for some elements $\left(y_{i}, x_{i_{1}} x_{i_{2}}, x_{i_{3}}\right)$ of $Y \times X^{3}$ and $\epsilon_{i} \in\{ \pm 1\}$. Since $\left|\theta\left(\left(y_{i}, x_{i_{1}} x_{i_{2}}, x_{i_{3}}\right)\right)\right| \leq 1$, we have $\left|\theta\left(c_{D^{\prime}, C^{\prime}}\right)\right| \leq t(F)$.

This theorem can be used to estimate the minimal triple point numbers of surface-links. For example, see Proposition 9.4 and Theorems 9.5 and 9.6 . 


\section{EXAMPLES AND APPLICATIONS}

First, we give some examples of quandle cocycles.

Example 9.1 (23]). Let $X$ be the order 6 trivial quandle $T_{6}=\left\{e_{1}, e_{1}^{\prime}, e_{2}, e_{2}^{\prime}, e_{3}, e_{3}^{\prime}\right\}$ and $\rho: X \rightarrow X$ be a good involution with $\rho\left(e_{i}\right)=e_{i}^{\prime}(i \in\{1,2,3\})$. Let $Y=\{e\}$, which is an $(X, \rho)$-set. Define a map $\theta: Y \times X^{3} \rightarrow \mathbb{Z}$ by

$$
\begin{aligned}
\theta= & \chi_{\left(e, e_{1}, e_{2}, e_{3}\right)}+\chi_{\left(e, e_{1}^{\prime}, e_{2}^{\prime}, e_{3}\right)}+\chi_{\left(e, e_{e}^{\prime}, e_{2}, e_{3}^{\prime}\right)}+\chi_{\left(e, e_{1}, e_{2}^{\prime}, e_{3}^{\prime}\right)} \\
& -\chi_{\left(e, e_{1}^{\prime}, e_{2}, e_{3}\right)}-\chi_{\left(e, e_{1}, e_{2}^{\prime}, e_{3}\right)}-\chi_{\left(e, e_{1}, e_{2}, e_{3}^{\prime}\right)}-\chi_{\left(e, e_{1}^{\prime}, e_{2}^{\prime}, e_{3}^{\prime}\right),}
\end{aligned}
$$

where $\chi_{(e, a, b, c)}$ is defined by $\chi_{(e, a, b, c)}(e, x, y, z)=1$ if $(e, x, y, z)=(e, a, b, c)$ and $\chi_{(e, a, b, c)}(e, x, y, z)=0$ otherwise. The linear extension $\theta: \mathbb{Z}\left(Y \times X^{3}\right) \rightarrow \mathbb{Z}$ is a 3 -cocycle. In [23] it is shown that the invariant $\Phi_{\theta}$ has information of the triple linking invariants [7] for oriented surface-links.

Example $9.2([23])$. Let $X$ be the order 2 trivial quandle $T_{2}=\left\{e_{1}, e_{2}\right\}$ and $\rho: X \rightarrow X$ be a good involution with $\rho(x)=x(x \in X)$. Let $Y=\{e\}$, which is an $(X, \rho)$-set. Define a map $\theta: Y \times X^{3} \rightarrow \mathbb{Z} / 2 \mathbb{Z}$ by

$$
\theta=\chi_{\left(e, e_{1}, e_{2}, e_{1}\right)} \text {. }
$$

The linear extension $\theta: \mathbb{Z}\left(Y \times X^{3}\right) \rightarrow \mathbb{Z} / 2 \mathbb{Z}$ is a 3-cocycle. In 23 it is shown that the invariant $\Phi_{\theta}$ has information of the mod 2 triple linking invariants 24 for surface-links which are not necessarily oriented or orientable.

Example 9.3. Let $X$ be the order 4 dihedral quandle $R_{4}$, in which we rename the elements $0,1,2,3$ by $e_{1}, e_{2}, e_{1}^{\prime}, e_{2}^{\prime}$, respectively. Let $\rho: X \rightarrow X$ be the antipodal map, i.e., $\rho\left(e_{i}\right)=e_{i}^{\prime}(i=1,2)$. Let $Y=\{e\}$, which is an $(X, \rho)$-set. Define a map $\theta: Y \times X^{3} \rightarrow \mathbb{Z}$ by

$$
\begin{aligned}
\theta= & +\chi_{\left(e, e_{1}, e_{2}, e_{1}\right)}+\chi_{\left(e, e_{1}^{\prime}, e_{2}^{\prime}, e_{1}\right)}+\chi_{\left(e, e_{1}^{\prime}, e_{2}, e_{1}^{\prime}\right)}+\chi_{\left(e, e_{1}, e_{2}^{\prime}, e_{1}^{\prime}\right)} \\
& -\chi_{\left(e, e_{1}^{\prime}, e_{2}, e_{1}\right)}-\chi_{\left(e, e_{1}, e_{2}^{\prime}, e_{1}\right)}-\chi_{\left(e, e_{1}, e_{2}, e_{1}^{\prime}\right)}-\chi_{\left(e, e_{1}^{\prime}, e_{2}^{\prime}, e_{1}^{\prime}\right)} \\
& -\chi_{\left(e, e_{2}, e_{1}, e_{2}\right)}-\chi_{\left(e, e_{2}^{\prime}, e_{1}^{\prime}, e_{2}\right)}-\chi_{\left(e, e_{2}^{\prime}, e_{1}, e_{2}^{\prime}\right)}-\chi_{\left(e, e_{2}, e_{1}^{\prime}, e_{2}^{\prime}\right)} \\
& +\chi_{\left(e, e_{2}^{\prime}, e_{1}, e_{2}\right)}+\chi_{\left(e, e_{2}, e_{1}^{\prime}, e_{2}\right)}+\chi_{\left(e, e_{2}, e_{1}, e_{2}^{\prime}\right)}+\chi_{\left(e, e_{2}^{\prime}, e_{1}^{\prime}, e_{2}^{\prime}\right)} .
\end{aligned}
$$

The linear extension $\theta: \mathbb{Z}\left(Y \times X^{3}\right) \rightarrow \mathbb{Z}$ is a 3 -cocycle.

Now we give some applications on minimal triple point numbers of surface-links. The following is obtained from Theorem 8.6

Proposition 9.4. Let $(X, \rho), Y$, and $\theta$ be the symmetric quandle, the $(X, \rho)$-set, and the 3-cocycle given in Example 9.3. Suppose that a surface-link $F$ has a diagram

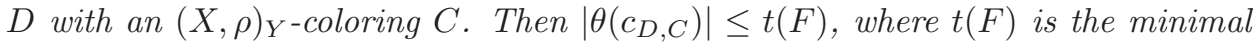
triple point number of $F$.

Let $M=M_{1} \cup M_{2}$ be a 2-component compact surface properly embedded in $\mathbb{R}^{3} \times[0,1]$ whose motion picture is given in Figure 22. The first still shows a trivial 2 -component link, which is equal to the last still. The second still is obtained from the previous still by a pair of Reidemeister moves of type II. The third is obtained by a pair of Reidemeister moves of type I. There are two saddle points between the third still and the fourth. The fifth still is obtained from the fourth by a pair of Reidemeister moves of type III, which are applied around the asterisked regions as in Figure 23. The sixth still is obtained by a pair of Reidemeister moves of type I, and the last still is obtained by a pair of Reidemeister moves of type II. 

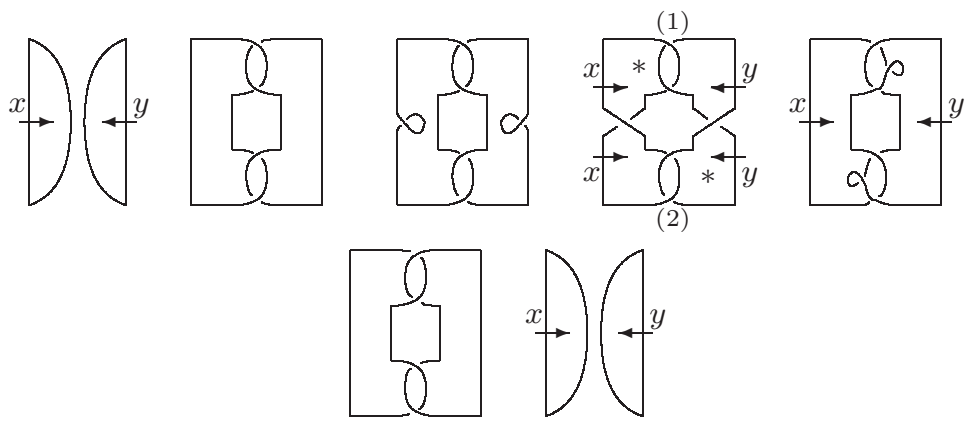

Figure 22. A motion picture of $M$
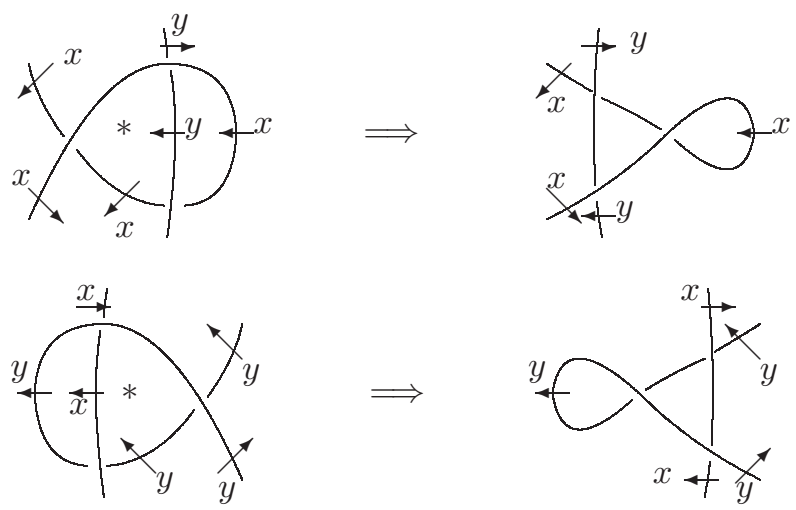

FiguRE 23. Reidemeister moves of type III

For a positive integer $n$, put

$$
M^{(n)}=\left\{x+(0,0,0, k) \in \mathbb{R}^{4}=\mathbb{R}^{3} \times \mathbb{R} \mid x \in M, k \in\{0,1, \ldots, n-1\}\right\},
$$

which is a properly embedded surface in $\mathbb{R}^{3} \times[0, n]$. Let $F=F^{(n)}$ be a closed surface in $\mathbb{R}^{4}=\mathbb{R}^{3} \times \mathbb{R}$ which is obtained from $M^{(n)}$ by attaching some disks trivially in $\mathbb{R}^{3} \times(-\infty, 0]$ and $\mathbb{R}^{3} \times[n, \infty)$. Then $F$ is a 2-component surface-link, and each component is a non-orientable surface. Satoh 24 proved that the minimal triple point number of $F$ is $2 n$ by a geometric argument on the normal Euler number. We can generalize this fact by the quandle cocycle invariant given in Example 9.3.

Theorem 9.5. Let $F=F^{(n)}$ be the surface-link described above. For any orientable surface-knot $K, t(F \# K) \geq 2 n$. Moreover, if $K$ is an orientable pseudo-ribbon surface-knot, then $t(F \# K)=2 n$. (A surface-knot $K$ is said to be pseudo-ribbon if $t(K)=0$.

Proof. Considering the motion picture of $M$ (Figure 22) to be a 1-parameter family of a classical link diagram, we have a broken surface diagram of $M$ with two triple points which correspond to the Reidemeister moves of type III in Figure 23. Combining $n$ copies of the broken surface diagram and capping off by trivial disks, we obtain a diagram of the surface-link $F$ with $2 n$ triple points, say $D$. Let $(X, \rho)$, $Y$, and $\theta$ be as in Example 9.3 Let $C$ be a coloring of $D$ whose restriction to 
each copy of the diagram of $M$ is as in Figure 22, where $x$ and $y$ are elements of $X$. Then $c_{D, C}=n(e, x, y, x)-n(e, y, x, y) \in C_{3}^{\mathrm{Q}, \rho}(X)_{Y}$. If $(x, y)=\left(e_{1}, e_{2}\right)$, then $\theta\left(c_{D, C}\right)=2 n$. By Proposition 9.4 $t(F) \geq 2 n$. Thus we have $t(F)=2 n$.

It is seen by Proposition 9.4 that $t(F \# K) \geq 2 n$ where $F \# K$ is a connected sum of $F$ and an orientable surface-knot $K$, since the colored diagram $(D, C)$ of $F$ can be extended uniquely to a colored diagram $\left(D^{\prime}, C^{\prime}\right)$ of $F \# K$ such that $c_{D, C}=c_{D^{\prime}, C^{\prime}} \in C_{3}^{\mathrm{Q}, \rho}(X)_{Y}$.

If $K$ is an orientable pseudo-ribbon surface-knot, then $F \# K$ has a diagram with $2 n$ triple points. Thus $t(F \# K)=2 n$.

The following was suggested by S. Satoh.

Theorem 9.6. For any positive integers $n_{1}$ and $n_{2}$ with $n_{1} \equiv n_{2}(\bmod 2)$, there is a surface-link $F_{1} \cup F_{2}$ such that $F_{i}(i=1,2)$ is the connected sum of $n_{i}$ copies of projective planes and $t\left(F_{1} \cup F_{2}\right)=2 \min \left\{n_{1}, n_{2}\right\}$.

Proof. Let $n=\min \left\{n_{1}, n_{2}\right\}$ and $F=F^{(n)}$ be the surface-link constructed above. Let $K$ be an unknotted orientable surface-knot of genus $\left(\max \left\{n_{1}, n_{2}\right\}-n\right) / 2$. As shown above, $t(F \# K)=2 n$. The components of $F \# K$ are non-orientable surfaces which are the connected sums of $n_{i}(i=1,2)$ copies of projective planes.

\section{ACKNOWLEDGMENTS}

The authors would like to thank Shin Satoh for helpful suggestions. This paper was completed while the second author visited Scott Carter and Masahico Saito. The authors would also like to thank them for their helpful comments.

\section{REFERENCES}

[1] N. Andruskiewitsch and M. Graña, From racks to pointed Hopf algebras, Adv. Math. 178 (2003), 177-243. MR:1994219 (2004i:16046)

[2] S. Asami and S. Satoh, An infinite family of non-invertible surfaces in 4-space, Bull. London Math. Soc. 37 (2005), 285-296. MR2119028 (2005k:57044)

[3] J. S. Carter, M. Elhamdadi, M. Graña and M. Saito, Cocycle knot invariants from quandle modules and generalized quandle cohomology, Osaka J. Math. 42 (2005), 499-541. MR2166720 (2006d:57017)

[4] J. S. Carter, D. Jelsovsky, S. Kamada, L. Langford and M. Saito, Quandle cohomology and state-sum invariants of knotted curves and surfaces, Trans. Amer. Math. Soc. 355 (2003), 3947-3989. MR 1990571 (2005b:57048)

[5] J. S. Carter, D. Jelsovsky, S. Kamada and M. Saito, Quandle homology groups, their Betti numbers, and virtual knots, J. Pure Appl. Algebra 157 (2001), 135-155. MR 1812049 (2002f:57010)

[6] J. S. Carter, S. Kamada and M. Saito, Geometric interpretations of quandle homology and cocycle knot invariants, J. Knot Theory Ramifications 10 (2001), 345-358. MR1825963 (2002h:57009)

[7] J. S. Carter, S. Kamada, M. Saito and S. Satoh, A theorem of Sanderson on link bordisms in dimension 4, Algebr. Geom. Topol. 1 (2001) 299-310. MR1834778 (2002d:57018)

[8] J. S. Carter and M. Saito, Knotted surfaces and their diagrams, Mathematical Surveys and Monographs, 55, Amer. Math. Soc., Providence, RI, 1998. MR1487374 (98m:57027)

[9] R. Fenn and C. Rourke, Racks and links in codimension two, J. Knot Theory Ramifications 1 (1992), 343-406. MR:1194995 (94e:57006)

[10] R. Fenn, C. Rourke and B. Sanderson, Trunks and classifying spaces, Appl. Categ. Structures 3 (1995), 321-356. MR 1364012 (96i:57023)

[11] R. Fenn, C. Rourke and B. Sanderson, The rack space, Trans. Amer. Math. Soc. 359 (2007), 701-740. MR2255194 (2007g:55013) 
[12] M. Iwakiri, Calculation of dihedral quandle cocycle invariants of twist spun 2-bridge knots, J. Knot Theory Ramifications 14 (2005), 217-229. MR2128511(2005k:57046)

[13] D. Joyce, A classifying invariant of knots, the knot quandle, J. Pure Appl. Algebra 23 (1982), 37-65. MR638121 (83m:57007)

[14] S. Kamada, Wirtinger presentations for higher dimensional manifold knots obtained from diagrams, Fund. Math.168 (2001), 105-112. MR1852735(2002g:57050)

[15] S. Kamada, Knot invariants derived from quandles and racks, Geom. Topol. Monogr. 4 (2002), 103-117. MR2002606 (2004g:57026)

[16] S. Kamada, Quandles with good involutions, their homologies and knot invariants, in: Intelligence of Low Dimensional Topology 2006, Eds. J. S. Carter et. al., pp. 101-108, World Scientific Publishing Co., 2007. MR2371714 (2009a:57042)

[17] R. A. Litherland and S. Nelson, The Betti numbers of some finite racks, J. Pure Appl. Algebra 178 (2003), 187-202. MR.1952425 (2004a:57006)

[18] S. Matveev, Distributive groupoids in knot theory (Russian), Mat. Sb. (N.S.) 119 (1982), 78-88; English translation: Math. USSR-Sb. 47 (1984), 73-83. MR672410 (84e:57008)

[19] T. Mochizuki, Some calculations of cohomology groups of finite Alexander quandles, J. Pure Appl. Algebra 179 (2003), 287-330. MR1960136 (2004b:55013)

[20] D. Roseman, Reidemeister-type moves for surfaces in four dimensional space, in: Knot Theory, Banach Center Publications 42 (1998), 347-380. MR1634466 (99f:57029)

[21] C. Rourke and B. Sanderson, There are two 2-twist spun trefoils, preprint, arxiv:math.GT/0006062:v1.

[22] C. Rourke and B. Sanderson, A new classification of links and some calculation using it, preprint, arxiv:math.GT/0006062:v2.

[23] K. Oshiro, Homology groups of trivial quandles with good involutions and triple linking numbers of surface-links, J. Knot Theory Ramifications, to appear.

[24] S. Satoh, Triple point invariants of non-orientable surface-links, Topology Appl. 121 (2002) 207-218. MR.1903691 (2003f:57048)

[25] S. Satoh, A note on the shadow cocycle invariant of a knot with a base point, J. Knot Theory Ramifications 16 (2007), 959-967. MR:2354269 (2008h:57023)

[26] S. Satoh and A. Shima, The 2-twist-spun trefoil has the triple point number four, Trans. Amer. Math. Soc. 356 (2004), 1007-1024. MR1984465 (2004k:57032)

[27] S. Satoh and A. Shima, Triple point numbers and quandle cocycle invariants of knotted surfaces in 4-space, New Zealand J. Math. 34 (2005), 71-79. MR2141479 (2006e:57031)

[28] M. Takasaki, Abstraction of symmetric transformations (Japanese), Tohoku Math. J. 49 (1943), 145-207. MR0021002 (9:8c)

Department of Mathematics, Hiroshima University, Hiroshima 739-8526, Japan

E-mail address: kamada@math.sci.hiroshima-u.ac.jp

Department of Mathematics, Hiroshima University, Hiroshima 739-8526, Japan

E-mail address: koshiro@iroshima-u.ac.jp 